\title{
Structural and dynamical properties predicted by reactive force fields simulations for four common pure fluids at liquid and gaseous non-reactive conditions
}

\author{
Tran Thi Bao Le and Alberto Striolo* \\ Department of Chemical Engineering, University College London, London WC1E 6BT \\ United Kingdom
}

David R. Cole

School of Earth Sciences, Ohio State University, Columbus, Ohio 43210

United States

\begin{abstract}
Four common pure fluids were chosen to elucidate the reliability of reactive force fields in estimating bulk properties of selected molecular systems: $\mathrm{CH}_{4}, \mathrm{H}_{2} \mathrm{O}, \mathrm{CO}_{2}$ and $\mathrm{H}_{2}$. The pure fluids are not expected to undergo chemical reactions at the conditions chosen for these simulations. The 'combustion' ReaxFF was chosen as reactive force field. In the case of water, we also considered the 'aqueous' ReaxFF model. The results were compared to data obtained implementing popular classic force fields. In the gas phase it was found that simulations conducted using the 'combustion' ReaxFF formalism yield structural properties in reasonable good agreement with classic simulations for $\mathrm{CO}_{2}$ and $\mathrm{H}_{2}$, but not for $\mathrm{CH}_{4}$ and $\mathrm{H}_{2} \mathrm{O}$. In the liquid phase 'combustion' ReaxFF simulations reproduce reasonably well the structure obtained from classic simulations for $\mathrm{CH}_{4}$, degrade for $\mathrm{CO}_{2}$ and $\mathrm{H}_{2}$, and are rather poor for $\mathrm{H}_{2} \mathrm{O}$. In the gas phase the simulation results are compared to experimental second virial coefficient data. The 'combustion' ReaxFF simulations yield second virial coefficients that are not sufficiently negative for both $\mathrm{CH}_{4}$ and $\mathrm{CO}_{2}$, and slightly too negative for $\mathrm{H}_{2}$. The 'combustion' ReaxFF parameterization induces too strong an effective attraction between water molecules, while the 'aqueous' ReaxFF yields a second virial coefficient for water that is in reasonable agreement with experiments. The 'combustion' ReaxFF parameterization yields acceptable self-diffusion coefficients for gas-phase properties of $\mathrm{CH}_{4}, \mathrm{CO}_{2}$ and $\mathrm{H}_{2}$. In the liquid phase the results are good for $\mathrm{CO}_{2}$, while the self-diffusion coefficient predicted for liquid $\mathrm{CH}_{4}$ is slower, and that predicted for liquid $\mathrm{H}_{2}$ is about 9 times faster than those expected based on classic simulations. The 'aqueous' ReaxFF parameterization yields good results for both the structure and the diffusion of both bulk liquid and bulk vapour water.
\end{abstract}




\section{INTRODUCTION}

Many classic (non-reactive) force fields have been developed to describe pure fluids and sometimes their mixtures. In these approaches chemical bonds are defined at the beginning of a simulation and constrained throughout. Each of the successful classic force fields was developed to match selected properties. For example, the TraPPE force fields were developed with the goal of reproducing vapor-liquid coexistence properties with the objective of allowing researchers to 'transfer' the force fields to molecules with similar chemistry [1-5]. While many thermodynamic and transport properties can be extracted from molecular simulations, arguably, the radial distribution function and the self-diffusion coefficients are fundamental properties that should be obtained reliably from a simulation. If this were not the case, estimating any thermodynamic and transport properties would not be straightforward.

While generally successful at predicting structural and transport properties of non-reactive systems, the classic models cannot describe systems undergoing chemical reactions in a molecular dynamics simulation. Reactive force fields have been developed for this purpose. The ReaxFF inventory of reactive force fields is attracting particular attention [6-7]. ReaxFF potentials provide an atomistic description of chemical reactions by allowing the formation and dissociation of chemical bonds. In addition, ReaxFF describes van der Waals and Coulombs interactions, thus comprehensively describing physical interactions in molecular systems. The force field parameters are typically optimized against a training set obtained from quantum mechanical calculations, which are essential for achieving a satisfactory description of the reactive events. ReaxFF parameter sets have been developed to accurately predict the characteristics and evolution of complex reactive systems such as the oxidation of hydrocarbons [8], the catalytic formation of nanotubes [9], surface catalytic chemistry [1011], and even electrochemical mechanisms [12-13]. Senftle et al. [6] recently reviewed the historical development of the ReaxFF approach. After the initial attempts to study reactive systems, now several parameterizations are available. Because we are interested in using the ReaxFF formalism to test our recent predictions regarding the effect of confinement on the equilibrium composition of the $\mathrm{CO}_{2}$ methanation reaction [14], we are particularly interested in the ReaxFF force fields developed to study combustion [8]. This parameterization is indicated as 'combustion' in what follows. However, we found that to simulate water it is preferable to implement the 'aqueous' ReaxFF parameterization [15], which is indicated as 'aqueous' in what follows. Raju et al. [15] adapted the aqueous parameterization to study water adsorption on titania, showing that mixed molecular and dissociative adsorption occurs, and suggesting that water molecules in the second adsorbed layer affect the equilibrium structure of adsorbed water. This is the parameterisation we implement, even though no titania surface is present in our systems.

Because of the ReaxFF success, attempts have been made to implement it to study structural, transport, and reactive properties of a fluid system, all within a single simulation. For example, Huang et al. [16] simulated water on various $\mathrm{TiO}_{2}$ surfaces, on which they studied the formation of hydrogen bonds, the layering of interfacial water, and the surface reactivity. The results demonstrated that $\mathrm{TiO}_{2}$ surfaces display different surface reactivity toward water dissociation. However, because the ReaxFF parameters are derived from quantum mechanical 
calculations, and have the clear objective of describing bond-forming and bond-breaking, one might not expect a priory that structural and transport properties are reproduced satisfactorily. This should not be surprising. For example, significant deviations are sometimes observed among results obtained for the same fluid at the same thermodynamic conditions when different classic force fields are implemented. For instance, Aimoli et al. [17] compared the ability of seven $\mathrm{CO}_{2}$ models (Cygan, EPM2, Zhang, etc.,) and three $\mathrm{CH}_{4}$ models (TraPPE, OPLS, SAFT- $\gamma$ ) to predict transport properties. Their results show that, e.g., the fully flexible models designated as Cygan and TraPPE-flex reproduce transport properties of $\mathrm{CO}_{2}$ with accuracy comparable to that obtained with rigid models (Zhang, EPM2, etc.), but underestimate some thermodynamic properties. Among the three rigid three-site models considered, the one developed by Zhang provides the best representation of $\mathrm{CO}_{2}$. Within the models tested to describe $\mathrm{CH}_{4}$, the OPLS model outperformed the single-site TraPPE model concerning viscosity and self-diffusion, whereas the single-site model provided the best estimation of thermodynamic properties and thermal conductivity.

Among the ReaxFF applications, Chenoweth et al. [8] derived parameters to describe gasphase hydrocarbon oxidation (i.e., combustion). We identify these parameters as 'combustion' ReaxFF in the remainder of this manuscript. The 'combustion' parameters were developed based on ReaxFF formalism for hydrocarbons [18], and they have been widely implemented by others. For instance, Page and Moghtaderi [19] investigated the chemical mechanism of low-temperature partial oxidation of methane. He et al. [20] combined 'combustion' ReaxFF and density functional theory to study the intrinsic mechanism of methane explosion. They further combined the 'combustion' ReaxFF simulations with ab initio MD calculations with particular attention to the substantial effect of water addition at different reaction stages on gas explosions [21]. Cheng et al. [22] developed an approach to accelerate dynamics while describing the chemical reaction rates and mechanisms for largescale complex reactions. The promising results of these studies suggest that the 'combustion' ReaxFF is a good candidate to study reactive events related to hydrocarbon compounds.

The goal of this manuscript is to determine whether the 'combustion' ReaxFF parameters are also able to reliably replicate structural and diffusion properties of the pure fluids, when they are not undergoing reactions. To test this, we selected four common pure fluids, $\mathrm{CH}_{4}, \mathrm{H}_{2} \mathrm{O}$, $\mathrm{CO}_{2}$ and $\mathrm{H}_{2}$, which are the main components in the reactive systems studied employing 'combustion' ReaxFF. In the case of water, Raju et al. [15] showed that the 'aqueous' parameterization yields radial distribution function and self-diffusion coefficient for bulk liquid water in excellent agreement with experimental data at $298 \mathrm{~K}$. We compare the properties predicted by simulations implementing models developed within the 'combustion' ReaxFF against those obtained when classic force fields are implemented instead. The criteria used to assess the suitability of the ReaxFF force fields to replicate the bulk properties of the pure fluids considered consist in the similarity between the results obtained from the two simulations (implementing classic or reactive force fields). When possible, the similarity between simulations and correspondent experimental data is also discussed. While data for the radial distribution functions for pure liquids are available, and used here, in the gas phase we use experimental second virial coefficient data. 
In the remainder of the manuscript we first provide an overview of the simulation approaches implemented, and a summary of the main features of the force fields chosen. We then compare the results obtained, in terms of structure and dynamics of pure fluids, in the gas and liquid phases. We close with generalized observations.

\section{METHODS and ALGORITHMS}

\section{Molecular Dynamics Simulations}

All of the molecular dynamics (MD) simulations were conducted using the Large-scale Atomic/Molecular Massively Parallel Simulator (LAMMPS) package, version 20160514 [2324]. All simulations were conducted at equilibrium conditions for the pure compounds. Below we provide details on the force field implemented. It should be noted that in the present manuscript the ReaxFF force fields are not trained on DFT datasets. We use parameters that were developed by others to study combustions and/or aqueous systems.

\section{Reactive Force Field - ReaxFF}

The ReaxFF parameterisation uses instantaneous bond orders, calculated from interatomic distances, to describe chemical reactivity during the reaction process [18]. The bond orders depend on sigma, pi, and double-pi bonds. The corresponding values are updated during the solution of the equations of motion at each iteration, which allows for the bonds to dynamically dissociate and form. In the ReaxFF formalism, connectivity-dependent interactions (e.g., valence and torsion-angles) are bond-order dependent, and energies and forces associated to these terms disappear upon bond dissociation. Non-bonded interactions (van der Waals and Coulomb) between atom pairs are calculated irrespective of molecular connectivity. To prevent excessive repulsions, the force field shields non-bonded interactions at short distances. A distance-corrected Morse potential is employed to describe van der Waals interactions and the Electron Equilibration Method (EEM) is implemented to describe electrostatic interactions. The total ReaxFF potential energy, $\mathrm{E}_{\mathrm{system}}$, is given by:

$$
\mathrm{E}_{\mathrm{system}}=\mathrm{E}_{\mathrm{bond}}+\mathrm{E}_{\mathrm{val}}+\mathrm{E}_{\mathrm{tors}}+\mathrm{E}_{\mathrm{over}}+\mathrm{E}_{\mathrm{under}}+\mathrm{E}_{\mathrm{lp}}+\mathrm{E}_{\mathrm{vdwaals}}+\mathrm{E}_{\text {coulomb }}
$$

The contributions to $E_{\text {system }}$ include bond energies ( $\left.E_{\text {bond }}\right)$, valence angle energies $\left(E_{\mathrm{val}}\right)$, torsion-angle energies $\left(\mathrm{E}_{\text {tors }}\right)$, energy contributions to penalize both over-coordination and (optionally) stabilize under-coordination of atoms $\left(\mathrm{E}_{\mathrm{over}}\right.$ and $\left.\mathrm{E}_{\mathrm{under}}\right)$, lone-pair energies $\left(\mathrm{E}_{\mathrm{lp}}\right)$, and non-bonded van der Waals $\left(\mathrm{E}_{\mathrm{vdWaals}}\right)$ and Coulomb $\left(\mathrm{E}_{\text {coulomb }}\right)$ interaction terms.

Although the conditions chosen for the simulated systems are such that no chemical reaction is expected to take place for $\mathrm{CH}_{4}, \mathrm{CO}_{2}, \mathrm{H}_{2}$ and gaseous $\mathrm{H}_{2} \mathrm{O}$, in liquid water proton transfer can occur. Both 'combustion' and 'aqueous' ReaxFF models yield a system composition in which $\mathrm{H}_{3} \mathrm{O}^{+}$molecules are $\sim 0.5 \%$ of the $\mathrm{H}_{2} \mathrm{O}$ molecules. Several other species are also identified in these simulations, with a combined concentration of up to $8 \%$ of the water molecules in the simulations. 


\section{Classic Force Fields - TraPPE, OPLS, EPM2, Zhang, SPC, SPC/E, Frost}

Several non-reactive force fields were implemented for comparison. The total potential energy in these simulations is determined by the sum of energy terms including short-range pairwise interactions, Coulombic long-range interactions, bond stretch, and angle bending terms [25]. The dispersive interactions are described using Lennard-Jones - type potentials. Whereas the ReaxFF uses all-atom structures to simulate various compounds, conventional force fields use molecular models with different morphologies and number of sites. We selected a few force fields to perform our comparison. The parameters of various nonreactive force fields used to conduct simulations are summarised in Table 1.

A large number of models have been proposed to calculate thermodynamic properties for methane and carbon dioxide in an extended range of state conditions. Based on the results reported by Aimoli et al. [17], we chose the TraPPE single-site model [1] and the fully flexible five-site OPLS model [26] for $\mathrm{CH}_{4}$ simulations. We implemented the rigid three-site models EPM2 [27], TraPPE [28], and the model proposed by Zhang [29] for $\mathrm{CO}_{2}$ simulations.

Many models have been proposed for water [30-35]. Most water models were designed to account for a set of selected properties. Vega et al. [36] reported the TIP4P/ice model yields the best description for densities of all ice phases whereas SPC/E, TIP4P, TIP4P/Ew and TIP5P models overestimate experimental ice densities. These authors also reported that TIP4P model reproduces the phase diagram of water better than SPC/E and TIP5P models. Vega et al. [37] compared several water models (TIP3P, TIP4P, TIP5P and TIP4P/2005) to assess their ability to predict ten different water properties (vapor-liquid equilibrium, critical temperature, surface tension, etc.). They concluded that the TIP5P/2005 is the model that best reproduces experimental data, except for the dielectric constant. While the TIP5P model does not seem adequate to describe reliably the water phase diagram, critical point, density of ices and water behavior at high pressure, it does predict the melting point, the dielectric constant, diffusivity and the maximum in the density of liquid water at room pressure better than the TIP4P model. The rigid nonpolarizable SPC/E model for water [32] was proposed to reproduce reliably the radial distribution function of bulk liquid water at $298 \mathrm{~K}$ as obtained by Soper et al. [38] using neutron scattering. Because we have used SPC/E in many of our prior publications, and because it yields an excellent structure for liquid water, we chose this model to simulate water in the present manuscript. The SPC/E model also provides acceptable estimates for the internal energy, the density and the diffusivity for water at ambient conditions [39]. The SPC rigid model [30] was chosen to simulate gas-phase water, because it reproduces the thermodynamic properties of supercritical water [40-41].

A wide variety of classic force fields are available to describe hydrogen [42-47]. In this work, the model reported by Frost et al. [47] was implemented. In this model the Lennard-Jones parameters for the hydrogen molecule are extracted from experimental data [48]. This model consists of a LJ neutral sphere, which is computationally efficient. 
TABLE 1. Force field parameters implemented in the classic simulations described in this manuscript. Dispersive interactions are described by 12-6 Lennard-Jones (LJ) potentials, while electrostatic interactions are described by Coulombic potentials.

\begin{tabular}{lllll}
\hline Force field & Site & $\boldsymbol{\sigma}(\mathbf{n m})$ & $\boldsymbol{\varepsilon} \mathbf{( k J / m o l )}$ & $\mathbf{q}(\mathbf{e})$ \\
\hline TraPPE-UA [1] & $\mathrm{CH}_{4}$ & 0.3730 & 1.23054 & 0 \\
OPLS [26] & $\mathrm{C}$ & 0.3500 & 0.27614 & -0.24 \\
& $\mathrm{H}$ & 0.2500 & 0.12552 & 0.06 \\
EPM2 [27] & $\mathrm{C}$ & 0.2757 & 0.23388 & 0.6512 \\
& $\mathrm{O}$ & 0.3033 & 0.66937 & -0.3256 \\
TraPPE-CO 2 [28] & $\mathrm{C}$ & 0.2800 & 0.22449 & 0.70 \\
& $\mathrm{O}$ & 0.3050 & 0.65684 & -0.35 \\
Zhang [29] & $\mathrm{C}$ & 0.27918 & 0.23983 & 0.5888 \\
& $\mathrm{O}$ & 0.3000 & 0.68724 & -0.2944 \\
SPC/E [32] & $\mathrm{O}$ & 0.3166 & 0.65020 & -0.8476 \\
& $\mathrm{H}$ & 0.0000 & 0.00000 & 0.4238 \\
SPC [30] & $\mathrm{O}$ & 0.3166 & 0.65020 & -0.8200 \\
& $\mathrm{H}$ & 0.0000 & 0.00000 & 0.4100 \\
Frost et al. [47] & $\mathrm{H}_{2}$ & 0.2958 & 0.30764 & 0 \\
\hline
\end{tabular}

\section{Algorithms}

The simulations are conducted within the canonical ensemble (constant number of molecules, constant volume and constant temperature), unless otherwise specified. Transport and structural features are determined following the Einstein relation [49] for the self-diffusion coefficients, and radial distribution functions for structural properties, respectively. In the gas phase, we extracted the second virial coefficients from the radial distribution functions obtained for the pure compounds [50]. Experimental second virial coefficients are available. All simulations using single-site, flexible and rigid models were performed implementing the Nosé-Hoover thermostat with a fixed temperature-damping factor of $100 \mathrm{fs}$. The velocityVerlet algorithm was used to integrate all molecular dynamics equations of motion. Each simulation was performed within periodic boundary conditions in a cubic box.

The temperatures, number of molecules, density and time steps used in the simulations are given in Table 2. The force field parameters implemented here for the various non-reactive simulations are reported in Table 3. In the case of ReaxFF simulations, several parameters are necessary to describe bond length and bond angle, as for example corrections due to bond order and hybridization are included. These parameters are not reported here for brevity. Note that the time step implemented was different for each simulation. We ensured that the time step was adequate for the ReaxFF simulations, and we then employed the same time step in the corresponding non-reactive simulations. The cutoff distance of interatomic interactions for all simulations using classic force fields was fixed at $14 \AA$. The particle mesh Ewald (PME) [51] method was used to treat the long-range Columbic interactions. The Lorentz- 
Berthelot mixing rule is applied to determine the Lennard-Jones parameters for unlike interaction from values of like components [52].

We used a 0.3 bond order cutoff for simulations implementing 'combustion' ReaxFF. The system temperature, total energy and structural properties oscillated around a constant value after $5 \mathrm{~ns}$ and $10 \mathrm{~ns}$ for liquid- and gas-phase simulations, respectively. These lengths of simulations (5 and $10 \mathrm{~ns}$ ) were therefore considered adequate equilibration periods. For comparison, when water was simulated on titania using the 'aqueous' ReaxFF parameterization, $0.1 \mathrm{~ns}$ were considered sufficient to equilibrate the systems [15]. After equilibration, a production run of $1 \mathrm{~ns}$ was conducted here to obtain averages for data analysis. During the production phase all atomic coordinates were recorded very 100 time steps.

TABLE 2. Thermodynamic conditions, number of molecules simulated, density and time step for each of the system investigated in this work.

\begin{tabular}{|c|c|c|c|c|c|c|c|}
\hline Fluid & $\begin{array}{c}\mathbf{P c}_{\mathbf{c}} \\
\text { (MPa) }\end{array}$ & $\begin{array}{l}\mathbf{T}_{\mathbf{c}} \\
(\mathbf{K})\end{array}$ & $\begin{array}{c}\rho_{c} \\
(\mathrm{~g} / \mathrm{l})\end{array}$ & $\begin{array}{c}\text { Temperature } \\
\text { (K) }\end{array}$ & $\begin{array}{l}\text { Molecules } \\
\text { simulated }\end{array}$ & $\begin{array}{c}\text { Density } \\
(\mathrm{g} / \mathrm{l})\end{array}$ & $\begin{array}{c}\text { Time } \\
\text { step (fs) }\end{array}$ \\
\hline \multirow[t]{2}{*}{$\mathrm{CH}_{4}$} & $4.5992^{\mathrm{a}}$ & $190.55^{a}$ & $162.6^{\mathrm{a}}$ & 150 & 440 & 449 & 0.25 \\
\hline & & & & 298 & 216 & 0.66 & 0.1 \\
\hline \multirow[t]{3}{*}{$\mathrm{CO}_{2}$} & $7.38^{\mathrm{b}}$ & $304.18^{b}$ & $468^{b}$ & 240 & 400 & 1,089 & 0.5 \\
\hline & & & & 273 & 500 & 1,105 & 0.5 \\
\hline & & & & 298 & 100 & 1.8 & 0.1 \\
\hline \multirow[t]{3}{*}{$\mathrm{H}_{2} \mathrm{O}$} & $22.064^{c}$ & $647.14^{\mathrm{c}}$ & $322^{c}$ & 298 & 550 & 1,000 & 0.25 \\
\hline & & & & 298 & 421 & 1,440 & 0.25 \\
\hline & & & & 500 & 100 & 2.2 & 0.1 \\
\hline \multirow[t]{2}{*}{$\mathrm{H}_{2}$} & $1.3^{\mathrm{d}}$ & $33.2^{\mathrm{d}}$ & $31.3^{\mathrm{d}}$ & 15.2 & 500 & 76 & 0.1 \\
\hline & & & & 77.5 & 100 & 0.3 & 0.1 \\
\hline
\end{tabular}

$\mathrm{T}_{\mathrm{c}}, \mathrm{P}_{\mathrm{c}}$ and $\rho_{\mathrm{c}}$ are experimental critical temperature, pressure and density, respectively.

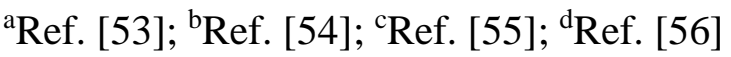

TABLE 3. Force fields parameters (bond lengths and angles) for the non-reactive force fields as implemented in this work.

\begin{tabular}{|c|c|c|c|c|c|c|c|}
\hline \multirow{2}{*}{ Model } & \multicolumn{4}{|c|}{ Bond length, $\AA$} & \multicolumn{3}{|c|}{ Angle, deg } \\
\hline & $\mathrm{C}-\mathrm{H}$ & $\mathrm{C}=\mathrm{O}$ & $\mathrm{O}-\mathrm{H}$ & $\mathrm{H}-\mathrm{H}$ & $\mathrm{H}-\mathrm{C}-\mathrm{H}$ & $\mathrm{O}=\mathrm{C}=\mathrm{O}$ & $\mathrm{H}-\mathrm{O}-\mathrm{H}$ \\
\hline TraPPE & & 1.161 & & & & 180 & \\
\hline Zhang & & 1.163 & & & & 180 & \\
\hline EPM2 & & 1.149 & & & & 180 & \\
\hline OPLS & 1.09 & & & & 107.8 & & \\
\hline $\mathrm{SPC} / \mathrm{E}$ & & & 1.0 & & & & 109.47 \\
\hline SPC & & & 1.0 & & & & 109.47 \\
\hline
\end{tabular}




\section{RESULTS}

\section{Structure}

Intermolecular pair correlation functions were obtained for $\mathrm{CH}_{4}, \mathrm{CO}_{2}, \mathrm{H}_{2}$ and $\mathrm{H}_{2} \mathrm{O}$ at liquid and gaseous conditions. The data obtained from the ReaxFF simulations are compared against those obtained, at the same thermodynamic conditions, using well-known classic nonreactive models. Below is a summary of our findings, which is followed by an analysis of the second virial coefficients obtained from our simulations in the gas phase.

Methane. The radial distribution functions, $g_{C C}, \mathrm{~g}_{C H}$, and $\mathrm{g}_{H H}$ are shown in Figure 1. Note that the TraPPE force field does not distinguish the $\mathrm{H}$ atoms of $\mathrm{CH}_{4}$, and therefore $\mathrm{g}_{C H}$ and $\mathrm{g}_{H H}$ cannot be computed for this model. The pair distribution functions are compared to those considered by Stassen as representative of experimental data [57]. It should be noted that Stassen conducted classic molecular dynamics simulations for liquid methane, although he employed parameters extracted from reverse Monte Carlo to reproduce neutron diffraction data for dense $\mathrm{CD}_{4}$ [58]. Considering liquid methane at $150 \mathrm{~K}$ [Fig. 1 (a-c)], the results show that implementing the 'combustion' ReaxFF yields peaks at shorter distances compared to what is predicted by the TraPPE and OPLS models, and also compared to experimental data. In addition, the heights of peaks obtained with 'combustion' ReaxFF are relatively similar to the corresponding ones obtained using OPLS.

The main features of $g_{C C}(r)$ are listed in Table 4 for all models. Although not shown in Fig. 1a, the peak positions and heights of an experimental $\mathrm{g}_{C C}(\mathrm{r})$ are included in Table 4. The $\mathrm{g}_{C C}(\mathrm{r})$ obtained for dense fluid methane [59] implementing the site-site exponential-6 model proposed by Williams [60] is illustrated in Fig. 1a. The exponential-6 potential of Williams has been demonstrated to be a good model for predicting the structure of liquid methane [58]. The first minimum in $g_{C C}$ for 'combustion' ReaxFF appears at $~ 5.5 \AA$, which is shifted by $\sim 0.2-0.3 \AA$ towards smaller distances than all the models as well as experiment. The number of neighbours, as estimated by the equation $n=4 \pi \int_{0}^{r_{m}} r^{2} g(r) \rho d r$, where $\mathrm{r}_{\mathrm{m}}$ is the first minimum of $g_{C C}(\mathrm{r})$, is 11.8 for 'combustion' ReaxFF compared to $~ 13$ from both OPLS and TraPPE simulations and $\sim 12.3 \AA$ from Williams model and experiment

The site-site pair distribution functions $\mathrm{g}_{\mathrm{CH}}(\mathrm{r})$ for 'experimental potential', 'combustion' ReaxFF, and OPLS model shown in Fig. 1b suggest that the first peak contains two maxima separated by the first minima. Although the shapes of $g_{C C}$ (Fig. 1a) and $g_{C H}$ peaks (Fig. 1b) are in close agreement among all the force fields, 'combustion' ReaxFF yields a slightly different structure for $\mathrm{g}_{H H}$ as compared to OPLS (Fig. 1c). In particular, a shoulder following the first peak is well formed from 'combustion' ReaxFF and experimental potential, but it is absent from the OPLS model results.

The observed differences are much more pronounced in the gas phase, which are illustrated in Fig. 1 (d-f). The 'combustion' ReaxFF peaks are shifted to shorter distances compared to data obtained from either TraPPE or OPLS models. In general, the magnitude of the $\mathrm{g}_{C C}, \mathrm{~g}_{C H}$ and $\mathrm{g}_{H H}$ peaks observed with 'combustion' ReaxFF is larger than that of the equivalent peaks 
obtained implementing the OPLS model, and smaller than that of the TraPPE model (TraPPE simulations are only available for $g_{C C}$ ). Moreover, there is a small hump at around $3.7 \AA$ in ReaxFF $\mathrm{g}_{C H}(\mathrm{r})$, which is absent in the OPLS dataset. Unfortunately, we could not find experimental data to directly compare the simulation results for gaseous $\mathrm{CH}_{4}$ at the conditions chosen here.

TABLE 4. Maxima and minima positions and heights of the carbon-carbon pair distribution functions $g_{C C}(r)$ of liquid methane with the investigated potential models. Corresponding plots are shown in Fig. 1a.

\begin{tabular}{|l|l|l|l|l|c|}
\hline \multicolumn{1}{|c|}{ Model } & 1st Max & 1st Min & 2nd Max & 2nd Min & 3rd Max \\
\hline 'Combustion' ReaxFF & $3.9 / 2.17$ & $5.5 / 0.70$ & $7.4 / 1.15$ & $9.1 / 0.91$ & $10.8 / 1.04$ \\
\hline OPLS & $4.0 / 2.30$ & $5.7 / 0.67$ & $7.6 / 1.18$ & $9.3 / 0.90$ & $11.0 / 1.05$ \\
\hline TraPPE & $4.0 / 2.84$ & $5.7 / 0.60$ & $7.7 / 1.26$ & $9.3 / 0.84$ & $11.0 / 1.09$ \\
\hline Exp-6 $^{\text {a }}$ & $4.1 / 2.35$ & $5.8 / 0.63$ & $7.7 / 1.17$ & $9.5 / 0.85$ & $11.3 / 1.05$ \\
\hline Exp $^{\text {b }}$ & $4.1 / 2.13$ & $5.8 / 0.65$ & $7.9 / 1.13$ & $9.5 / 0.87$ & \\
\hline
\end{tabular}

${ }^{a}$ The simulation results for the exponential-6 model were taken from Table 3 (model H) from Stassen [57].

${ }^{b}$ Data from Stassen [57], as obtained from a potential model adjusted to reproduce experimental neutron diffraction data for $\mathrm{CD}_{4}$. 

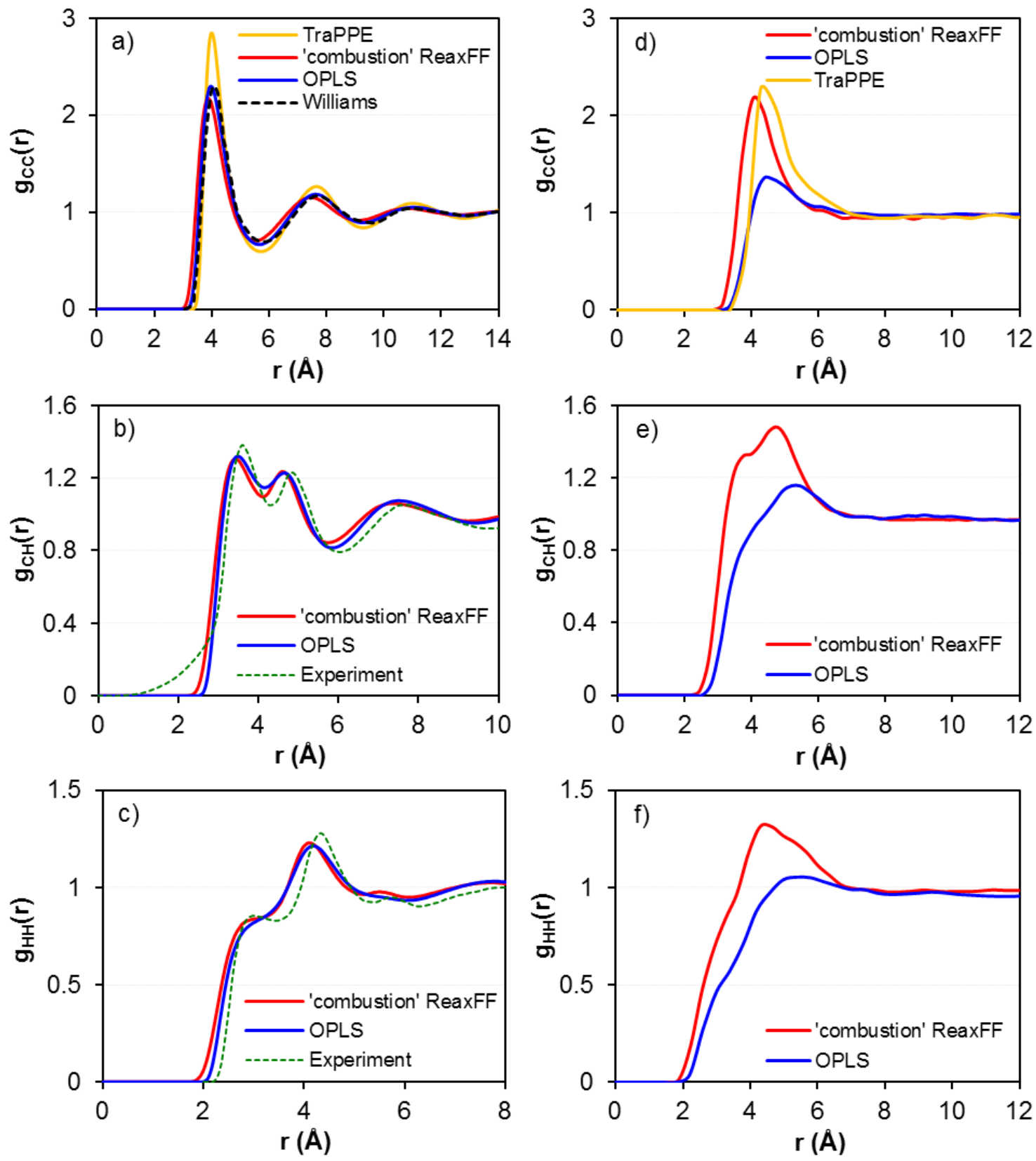

Figure 1. Site-site radial distribution functions for $\mathrm{CH}_{4}$ at $150 \mathrm{~K}$ and $\rho=449 \mathrm{~g} / \mathrm{l}(\mathrm{a}, \mathrm{b}$ and $\mathrm{c}$ ), and at $298 \mathrm{~K}$ and $\rho=0.66 \mathrm{~g} / \mathrm{l}(\mathrm{d}$, e and $\mathrm{f}$ ). Note that the TraPPE force field is united atom, and as such it does not differentiate the $\mathrm{H}$ atoms in $\mathrm{CH}_{4}$. 

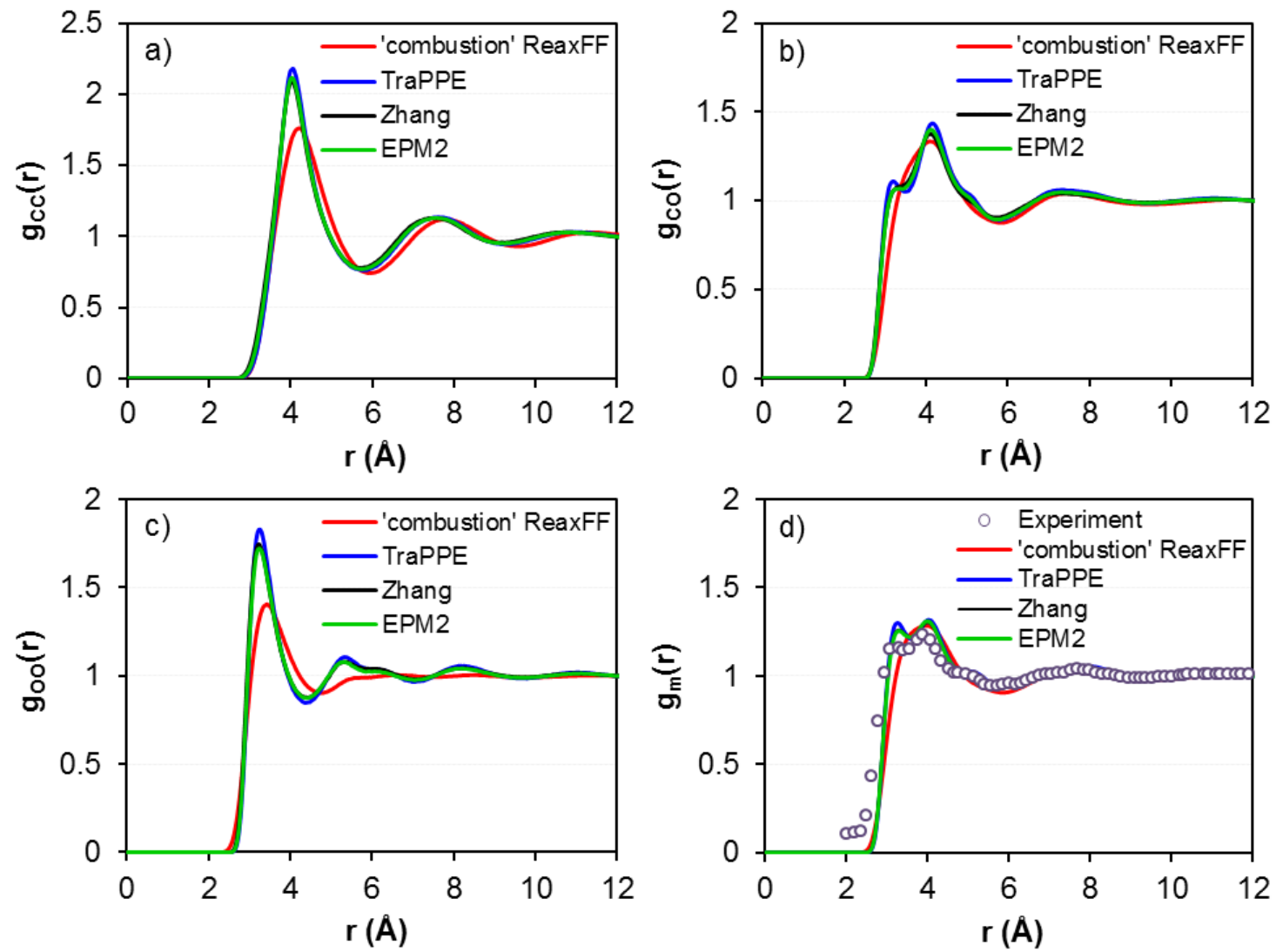

Figure 2. Site-site radial distribution functions ( $\mathrm{a}, \mathrm{b}$ and $\mathrm{c})$ and neutron weighted radial distribution functions for $\mathrm{CO}_{2}$ at $240 \mathrm{~K}$ and $\rho=1,089 \mathrm{~g} / \mathrm{l}$.

Carbon dioxide. Radial distribution functions obtained for $\mathrm{CO}_{2}$ are shown in Fig. 2 and Fig. 3. The results for liquid $\mathrm{CO}_{2}$ (Fig. 2) show that the $\mathrm{g}_{C C}(\mathrm{r})$ peaks from 'combustion' ReaxFF simulations are shifted to slightly larger distances by about $0.2 \AA$ compared to data obtained implementing classical force fields. The height of the first 'combustion' ReaxFF peak is lower, whereas the second and the third peaks are similar to those obtained by implementing the Zhang, EPM2 and TraPPE force fields. The number of molecules within the firstneighbour shell is $\sim 13$ for 'combustion' ReaxFF, as opposed to $\sim 12$ for the other three classic force fields.

Data for $\mathrm{g}_{C O}(\mathrm{r})$ are considerably different when 'combustion' ReaxFF is compared to the classical force fields wherein the first 'combustion' ReaxFF $g_{C O}(r)$ peak is a singlet, whereas the classic force fields yield a peak at $\sim 3.2 \AA$ as well as one at $\sim 4.1 \AA$. Also note that 'combustion' ReaxFF yields a second $\mathrm{g}_{C O}(\mathrm{r})$ peak weaker and shifted to slightly larger distances compared to that computed using classical force fields. Regarding $\mathrm{g} o o(\mathrm{r})$, 'combustion' ReaxFF yields a somewhat broader first peak and shifted to slightly larger distances than the corresponding classical simulations results. It should also be noted that the local structure beyond the first peak is missing in the 'combustion' ReaxFF goo(r).

To compare the simulated liquid structure of $\mathrm{CO}_{2}$ against experiments, the sum of three radial distribution functions as neutron-weighted distribution function $\mathrm{g}_{\mathrm{m}}(\mathrm{r})$ was computed [61]: 


$$
\mathrm{g}_{\mathrm{m}}(\mathrm{r})=0.403 \mathrm{~g}_{O O}(\mathrm{r})+0.464 \mathrm{~g}_{C O}(\mathrm{r})+0.133 \mathrm{~g}_{C C}(\mathrm{r})
$$

As shown in Fig. 2d, a split of the first peak is observed in the neutron-weighted distribution function obtained from classical models, which qualitatively reproduces the experimental data. This feature almost disappears from the simulation data extracted from the reactive simulations conducted with the 'combustion' ReaxFF parameters.
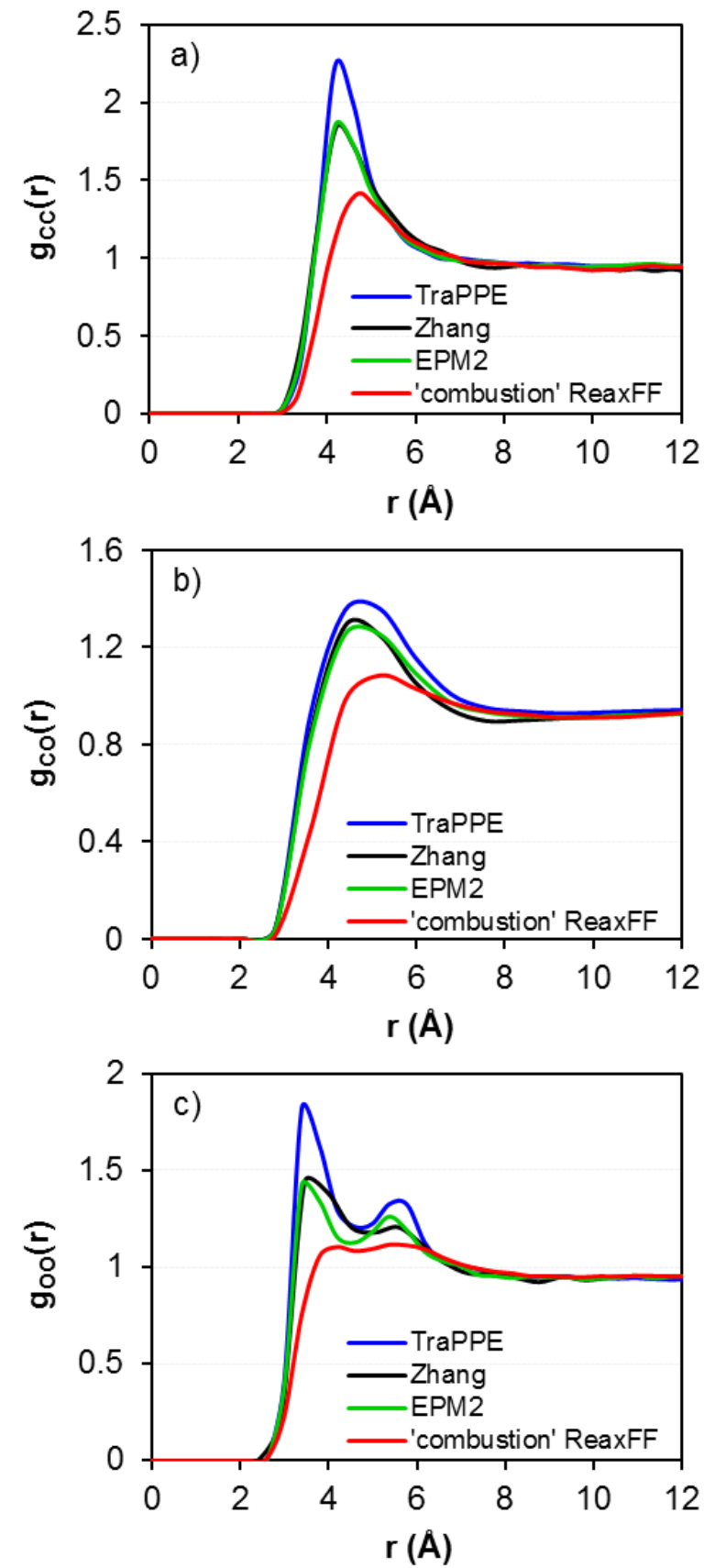

Figure 3. Site-site radial distribution functions for $\mathrm{CO}_{2}$ at $298 \mathrm{~K}$ and $\rho=1.8 \mathrm{~g} / \mathrm{l}$. 
The characteristics of the site-site pair distribution functions for gaseous $\mathrm{CO}_{2}$ at $298 \mathrm{~K}$ are displayed in Fig. 3. All the $\mathrm{g}_{C C}(\mathrm{r})$ from classical models produce a single peak at $\sim 4.2 \AA$. The single peak obtained from the 'combustion' ReaxFF simulations has lower intensity and is shifted by about $0.5 \AA$ to longer distances. The $\mathrm{g}_{C O}$ peak obtained from 'combustion' ReaxFF appears at larger distances and it is broader than the equivalent peaks obtained implementing TraPPE, Zhang and EPM2 models. With respect to goo(r), the 'combustion' ReaxFF yields two broad peaks at $4.2 \AA$ and $5.8 \AA$, which differ in both intensity and location compared with the two correspondent peaks predicted by classic models.

Water. We observed an unrealistic phase separation when implementing 'combustion' ReaxFF to simulate liquid water at ambient conditions. Therefore, we concluded that the 'combustion' ReaxFF parameterization is not adequate to simulate liquid water at ambient conditions, at least without further training of the parameters. Additional simulations were conducted under the NPT ensemble to determine the density of liquid water at ambient conditions according to the 'combustion' ReaxFF force field. In these simulations temperature and pressure were controlled by implementing the Nosé-Hoover thermostat and barostat, respectively. The density of $\sim 1400 \mathrm{~g} / \mathrm{l}$ was obtained, and used for subsequent simulations for liquid water within the NVT ensemble even though this density is clearly unrealistic. The structure of liquid water estimated using 'combustion' ReaxFF was then compared to that obtained from classic simulations that implement the SPC/E model. The results are presented below for completeness.

In Fig. 4 we show gOO, $\mathrm{g}_{O H}$ and $\mathrm{g}_{H H}$ radial distribution functions determined from 'combustion' ReaxFF simulations and SPC/E or SPC (liquid and gaseous water, respectively). The shapes and peak positions of all intermolecular pair correlation functions from 'combustion' ReaxFF simulations are quite different compared to those predicted by the classical force fields, which is in part due to the higher density at which some of these simulations were conducted compared to that expected for bulk liquid water. We conclude that the 'combustion' ReaxFF is not appropriate for predicting the structural properties of liquid water. Even the gas-phase simulations showed significant aggregation of water when the 'combustion' ReaxFF model was implemented, which yields very pronounced peaks in the radial distribution functions considered.

As described by Senftle et al. [6], the combustion parameterisation was developed to study hydrocarbon oxidation, while the aqueous parameterization was developed to study aqueous systems. We refer here to the latter ReaxFF parameterization as 'aqueous', and we implement the parameters developed by Monti et al. [62] The results from the 'aqueous' ReaxFF parameterisation are in excellent agreement with those obtained from the classical force fields for both liquid and gaseous phases. They are also in reasonable agreement with experimental data, as shown in Fig. 4. Considering liquid water, both SPC/E model and 'aqueous' ReaxFF yield almost the same positions and magnitudes for the first and the third peaks in goo $(\mathrm{r})$, both of which are in good agreement with experiments. The second peak for 'aqueous' ReaxFF is shifted by about $0.2 \AA$ towards smaller distance compared to data obtained from both SPC/E simulations and experiment. 
The results for $\mathrm{g}_{\mathrm{OH}}(\mathrm{r})$ are related to the structure of the hydrogen bond network formed by neighbouring water molecules. The first peak from both SPC/E and 'aqueous' ReaxFF simulations for liquid water appears at $\sim 1.8 \AA$, and the second peak appears at $\sim 3.3 \AA$ for both 'aqueous' ReaxFF and SPC/E simulations. The positions and most of the magnitudes of all the $\mathrm{g}_{\mathrm{OH}}$ peaks obtained from both 'aqueous' ReaxFF and SPC/E simulations are in agreement with experimental $\mathrm{g}_{\mathrm{OH}}$ peaks. A pronounced difference is however found in the magnitude of the first peak. Integrating the radial distribution functions, we calculated that approximately 2 hydrogen atoms are found at a distance lower than $\sim 2.4 \AA$ from each oxygen atom for both SPC/E and 'aqueous' ReaxFF simulations.

Two well-defined peaks in $\mathrm{g}_{H H}(\mathrm{r})$ are found at $\sim 2.4 \AA$ and $3.8 \AA$ for both 'aqueous' ReaxFF and SPC/E simulations that are in reasonable agreement with experiments, further suggesting that the 'aqueous' ReaxFF is suitable for describing the structure of liquid water.

Regarding water vapour, our simulation results, Fig. 3(d, e, f), further confirm that the 'combustion' ReaxFF parameterization is inadequate. On the other hand, our results suggest that the 'aqueous' ReaxFF parameterization is in good agreement with SPC model. Peak positions and heights of the $\mathrm{g}_{O O}(\mathrm{r})$ obtained from both 'aqueous' ReaxFF and SPC simulations are similar. The $\mathrm{g}_{H H}(\mathrm{r})$ and $\mathrm{g}_{O H}(\mathrm{r})$ have almost identical peak positions for both force fields, albeit 'aqueous' ReaxFF simulations yield smaller intensities than classic simulations implementing the SPC model. 

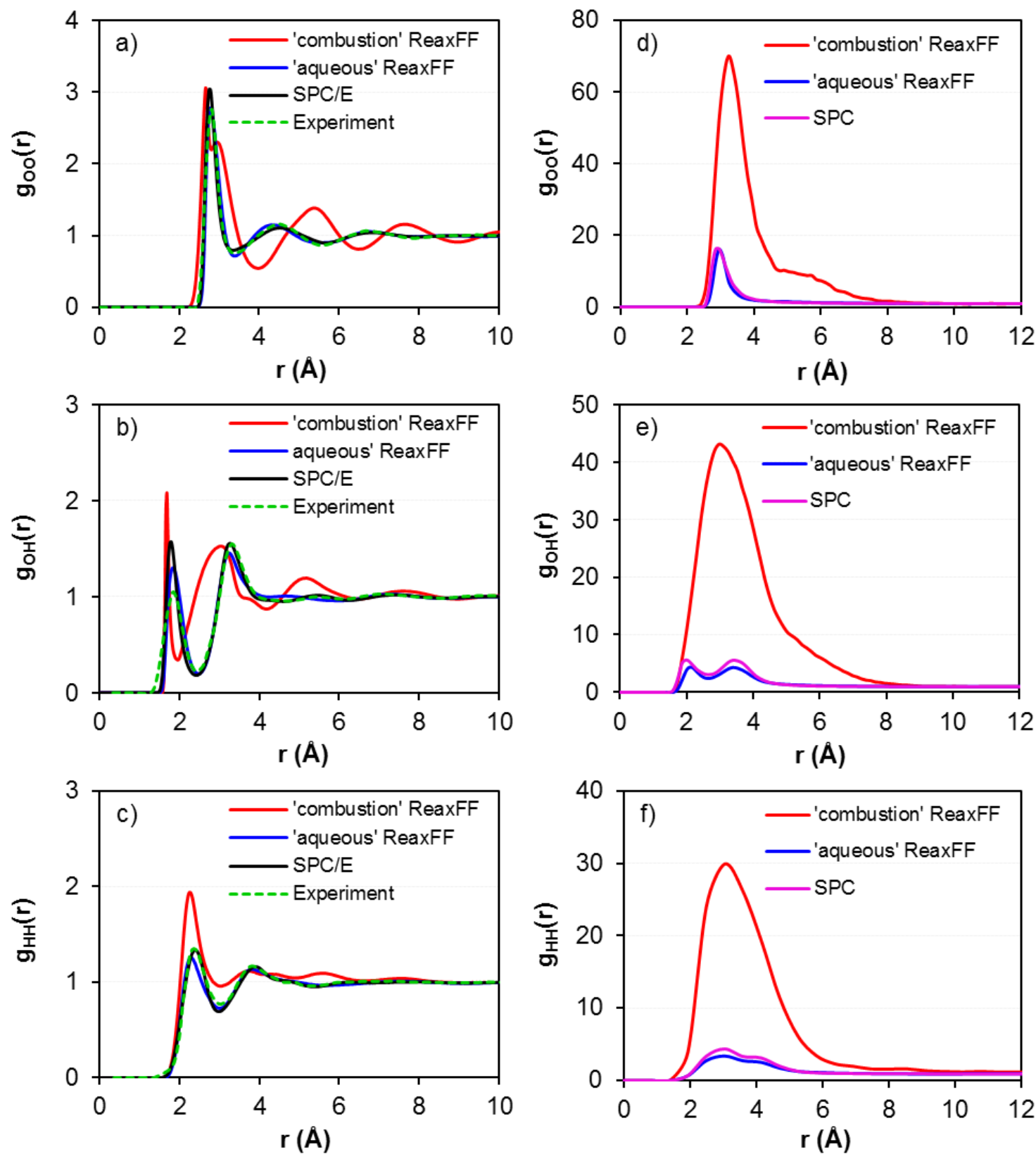

Figure 4. Site-site radial distribution functions for $\mathrm{H}_{2} \mathrm{O}$ at $298 \mathrm{~K}$ (a, b and c), and at $500 \mathrm{~K}$ (d, e and f). The density for 'combustion' ReaxFF simulations in panels $a, b$ and $c$ is of $1,440 \mathrm{~g} / \mathrm{l}$, and of $2.2 \mathrm{~g} / \mathrm{l}$ in panels $\mathrm{d}$, e and $\mathrm{f}$. All other thermodynamic conditions are reported in Table

2. Neutron diffraction experimental data from Soper et al. [38] are also shown.

To investigate the reason why the 'combustion' ReaxFF parameterisation fails in describing the structure of water, the simulation results were analysed at various run times (see Fig. 5). The first important observation is that the structure of water evolves during this analysis, hence equilibrium is not yet reached. This is perhaps not surprising, since these simulations are only 90 ps long, but it should be made clear to avoid confusion. It was found that although $\mathrm{g}_{O O}(\mathrm{r})$ maintains the same shape over time (Fig. 5(a-c)), $\mathrm{g}_{O H}(\mathrm{r})$ and $\mathrm{g}_{H H}(\mathrm{r})$ change over time. As seen in Fig. 5(d-f), g OOH$_{(\mathrm{r})}$ displays two well-defined peaks, one at $2.1 \AA$ and 
one at $3.6 \AA$, after 20 ps. The two peaks gradually vanish after 40 ps, and merge after 90 ps. Similarly, during the initial 20 ps, $g_{H H}$ (Fig. 4(g-i)) yields two peaks (at $3.0 \AA$ and at $4.1 \AA$ ). The second of these peaks is strongly reduced as the simulations progress. Although the 'combustion' ReaxFF seems able to yield a reasonable gas-phase water structure at short simulation times before equilibrium is achieved, after $\sim 90$ ps water molecules condense yielding a large cluster. This suggests that the attraction between 'combustion' ReaxFF water molecules in the gas phase is too strong.
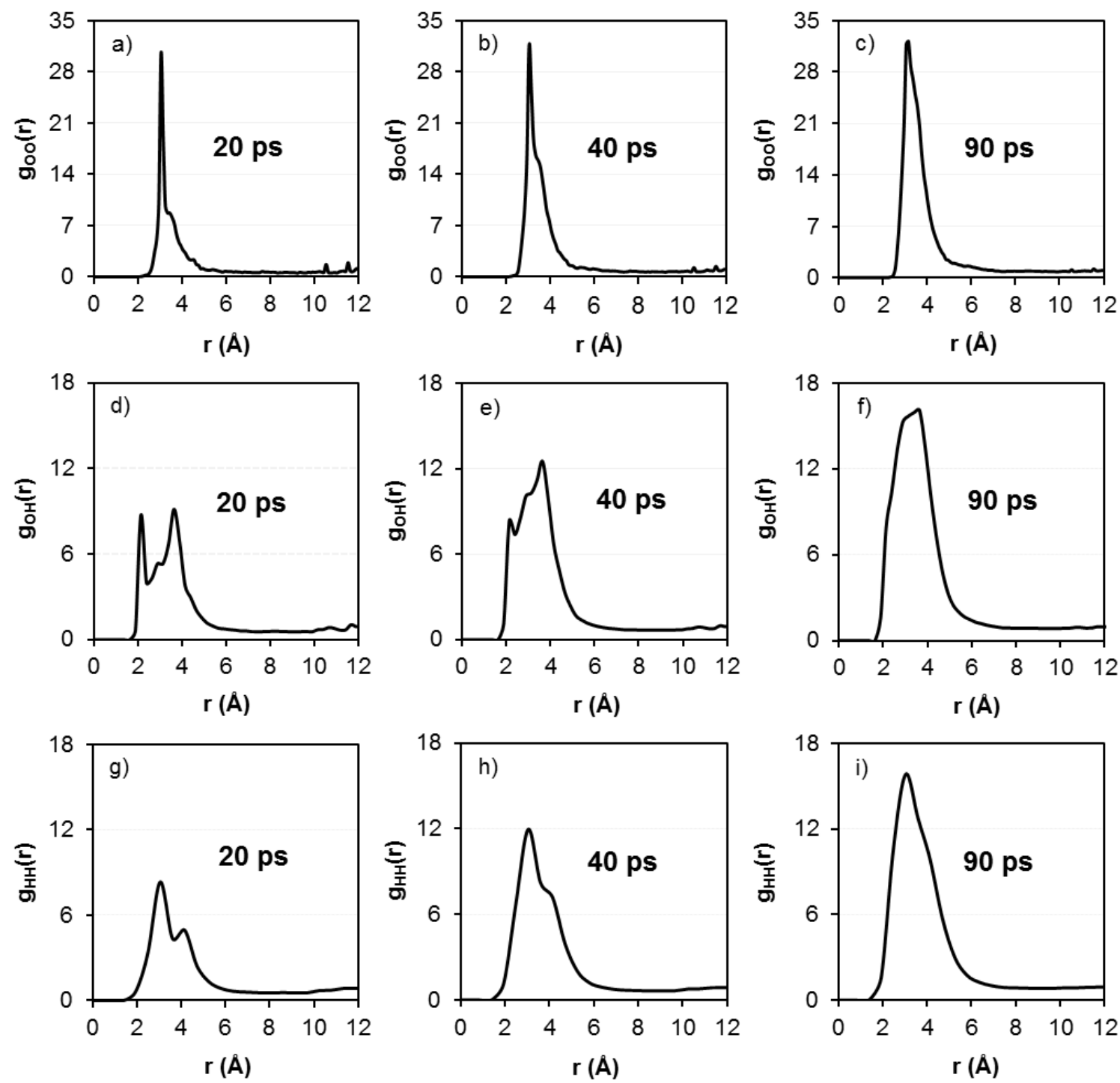

Figure 5. Site-site radial distribution functions of gas-phase water molecules as a function of time: $\mathrm{g}_{O O}(\mathrm{r})(\mathrm{a}-\mathrm{c}), \mathrm{g}_{O H}(\mathrm{r})(\mathrm{d}-\mathrm{f})$, and $\mathrm{g}_{H H}(\mathrm{r})(\mathrm{g}-\mathrm{i})$.

Hydrogen. In the case of $\mathrm{H}_{2}$, the 'combustion' ReaxFF parameterisation describes both atoms, while the classical force field is a united-atom approach. To compare the data, we consider the centre of mass of $\mathrm{H}_{2}$, and we compute the resultant $\mathrm{g}_{\mathrm{H} 2-\mathrm{H} 2}$ with the results shown in Fig. 6. Regarding liquid $\mathrm{H}_{2}$, the first peak position $(\sim 3.2 \AA)$ is quite similar in both models, but 'combustion' ReaxFF yields second, third and fourth peak positions at shorter 
distances and greater intensities compared to the non-reactive simulations. The number of $\mathrm{H}_{2}$ first neighbours within the first minimum in the pair correlation function (at $4.4 \AA$ ) is $\sim 8$ for 'combustion' ReaxFF. Classical simulations yield instead $\sim 9$.

For comparison purposes, in lieu of experimental data for hydrogen, we used radial distribution functions of liquid 'para-hydrogen' as obtained from correlated density-matrix (CDM) theory [63] and the path-integral-centroid-molecular-dynamics (PICMD) approach [64]. These theoretical results are often considered as reference data for hydrogen. Our results show a large difference in peaks positions and heights between both 'combustion' ReaxFF and classic simulations predictions and CDM or PICMD data for liquid hydrogen. It should be pointed out, however, that the Frost model was derived to study the gas phase of hydrogen, but it neglects quantum effects. It is likely that the difference between simulation results achieved when the Frost model or the 'combustion' ReaxFF models are implemented as opposed to predictions from the CDM or PICMD models are due to the fact that both CDM and PICMD methods incorporate quantum effects.

Both classical and reactive force fields exhibit a similar peak position at $\sim 3.4 \AA$ in the gas phase. However, the single peak predicted from 'combustion' ReaxFF is slightly higher and broader than when implementing the model described by Frost et al. [47] No difference is detected between the two force fields at distances larger than $6 \AA$.
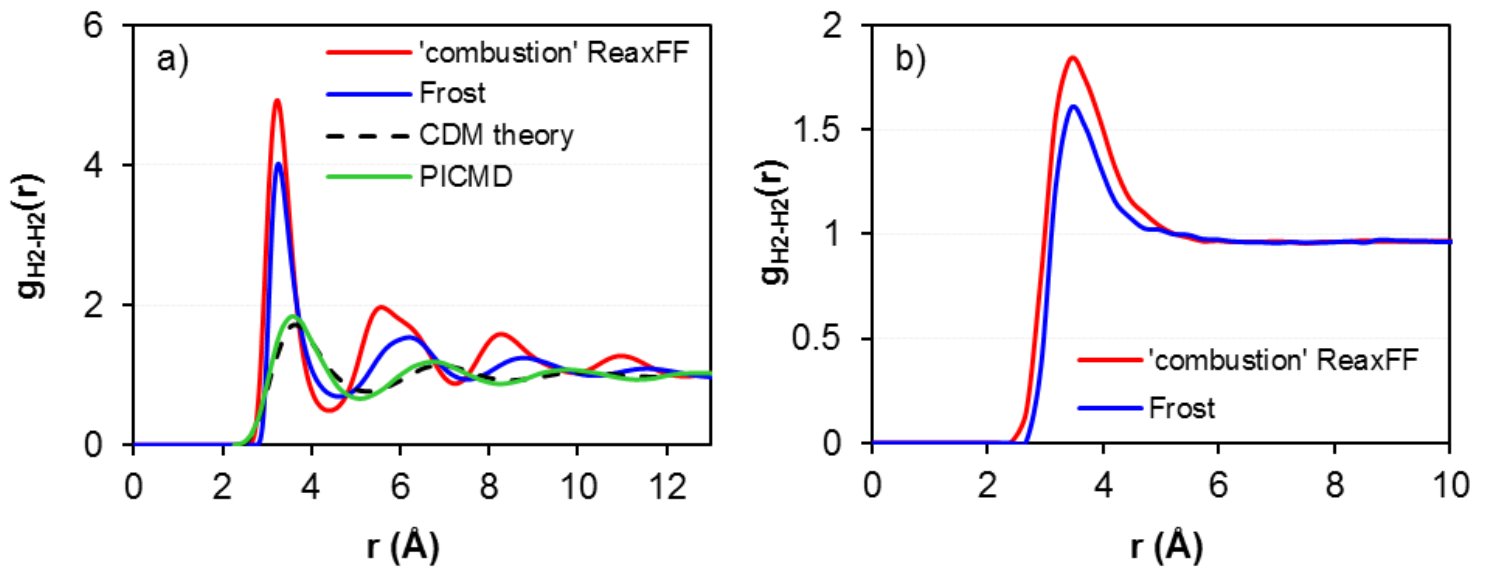

Figure 6. Site-site radial distribution functions for $\mathrm{H}_{2}$ at $15.2 \mathrm{~K}$ and $\rho=76 \mathrm{~g} / \mathrm{l}$ (a) and at 77.5

$\mathrm{K}$ and $\rho=0.3 \mathrm{~g} / \mathrm{l}(\mathrm{b})$. The result of CDM theory reported by Lindenau et al. [63] was computed for liquid 'para-hydrogen' at $16 \mathrm{~K}$ and $\rho=0.021 \AA^{-3}$.

\section{Second Virial Coefficients}

While it is problematic to directly compare the simulated radial distribution functions in the gas phase to experimental data, experimental data are available for the second virial coefficient, $\mathrm{B}_{2}$, which is a fundamental thermodynamic quantity. Several approaches can be 
employed to calculate $\mathrm{B}_{2}$ for either pure compounds or mixtures. The Mayer sampling method, based on free energy perturbation approaches [65], was implemented to calculate virial coefficients for a variety of potentials [66-67]. $\mathrm{B}_{2}$ for small alkanes and inert gases can be extracted from the simulated pair correlation functions [68-69] or from analytic equations of state [70-71]. Here we integrate the radial distribution functions [50]:

$$
\mathrm{B}_{2}(\mathrm{~T})=-2 \pi \int_{0}^{\infty} \mathrm{r}^{2}[\mathrm{~g}(\mathrm{r})-1] \mathrm{dr}
$$

In Eq. (3) $r$ is the distance between the centres of mass of two molecules and $g(r)$ is the radial distribution function. The second virial coefficients obtained from our simulations, as well as the corresponding experimental values, are reported in Table 5. In all cases, the results from ReaxFF simulations differ from those obtained using non-reactive force fields.

In the case of methane, the results suggest that the TraPPE force field yields too strong effective attractions compared to experimental observations (the more negative $\mathrm{B}_{2}$ is, the more attractive the effective interactions are), while the OPLS force field is not attractive enough. The 'combustion' ReaxFF parameters yield an effective interaction between $\mathrm{CH}_{4}$ molecules that is also too attractive compared to experiments. It is concluded that a realistic $\mathrm{g}_{\mathrm{CC}}$ would be in between that obtained for the OPLS and that obtained for the 'combustion' ReaxFF simulations.

In the case of $\mathrm{CO}_{2}$, none of the simulation results are consistent with the experimental data. The TraPPE force fields are those that yield data in closest agreement with experiments, while all the others, including the 'combustion' ReaxFF, do not yield sufficiently attractive interactions between $\mathrm{CO}_{2}$ molecules in the gas phase.

In the case of water, the 'combustion' ReaxFF parameters yield an unrealistically strong attraction, as already discussed in Figure 5. The 'aqueous' ReaxFF simulations yield a $B_{2}$ that is in closer agreement with experimental data than the SPC non-reactive simulations, although all simulations predict an effective attraction that is too strong compared to experiments.

In the case of hydrogen, both non-reactive and 'combustion' ReaxFF simulations yield $\mathrm{B}_{2}$ data that are slightly too attractive, yet in reasonable agreement with experimental data. 
TABLE 5. Second virial coefficients for gas-phase molecules estimated from our simulations. For comparison, experimental data are also reported.

\begin{tabular}{|c|c|c|c|c|}
\hline \multirow{2}{*}{ Molecule } & \multirow{2}{*}{ Temperature (K) } & \multirow{2}{*}{ Force field } & \multicolumn{2}{|c|}{ Second virial coefficient $\left(\mathrm{cm}^{3} / \mathrm{mol}\right)$} \\
\hline & & & This study & Experiment \\
\hline \multirow{3}{*}{$\mathrm{CH}_{4}$} & \multirow{3}{*}{298} & TraPPE & $-115 \pm 14$ & \multirow{3}{*}{$-43.25^{\mathrm{a}}$} \\
\hline & & OPLS & $-20 \pm 6$ & \\
\hline & & 'combustion' ReaxFF & $-75 \pm 5$ & \\
\hline \multirow{4}{*}{$\mathrm{CO}_{2}$} & \multirow{4}{*}{298} & TraPPE & $-100 \pm 20$ & \multirow{4}{*}{$-121.8^{\mathrm{b}}$} \\
\hline & & Zhang & $-56 \pm 17$ & \\
\hline & & EPM2 & $-70 \pm 19$ & \\
\hline & & 'combustion' ReaxFF & $-16 \pm 5$ & \\
\hline \multirow{3}{*}{$\mathrm{H}_{2} \mathrm{O}$} & \multirow{3}{*}{500} & SPC & $-390 \pm 45$ & \multirow{3}{*}{$-171.97^{\mathrm{c}}$} \\
\hline & & 'aqueous' ReaxFF & $-235 \pm 32$ & \\
\hline & & 'combustion' ReaxFF & $-5600 \pm 50$ & \\
\hline \multirow{2}{*}{$\mathrm{H}_{2}$} & \multirow{2}{*}{77.5} & Frost & $-13 \pm 6$ & \multirow{2}{*}{$-11.1^{\mathrm{d}}$} \\
\hline & & 'combustion' ReaxFF & $-25 \pm 7$ & \\
\hline
\end{tabular}

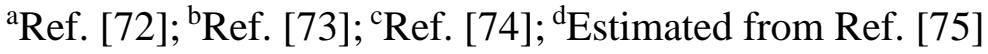

\section{Self-Diffusion Coefficients}

The self-diffusion coefficients were calculated from the mean square displacements by implementing the Einstein equation [49]:

$$
\lim _{t \rightarrow \infty}\left\langle\left|r_{i}\left(t^{\prime}+t\right)-r_{i}\left(t^{\prime}\right)\right|^{2}\right\rangle=6 D_{s} t
$$

In Eq. (4), $r_{i}(t)$ and $r_{i}\left(t^{\prime}\right)$ are the positions of molecule $i$ at time $t$ and at the time origin $t^{\prime}$, respectively. In these calculations, after the systems are equilibrated, the simulations are conducted for up to $2 \mathrm{~ns}$. The mean square displacements are calculated from at least 5, and sometimes above 10 origins, separated by at least 200 ps but no more than 500 ps. The molecular simulation results of self-diffusion coefficients for all models are compiled in Table 6, where we also provide experimental data for comparison. It should be remembered that the simulation box size has a strong effect on simulated self-diffusion coefficients. For example, Yeh and Hummer simulated water and a simple Lennard-Jones fluid [76]. They found that the self-diffusion coefficient increases with the size of the simulation box. They proposed an analytical correction, proportional to $\mathrm{N}^{-1 / 3}$, where $\mathrm{N}$ is the number of molecules in the system, to extrapolate the simulation results to the thermodynamic limit. Because of computing-power limitations, we quantified system-size effects only for liquid $\mathrm{CO}_{2}$ at $273 \mathrm{~K}$. When the number of molecules was increased from 500 to 1,000, and 1,500, the selfdiffusion coefficient obtained with the TraPPE force field was found to increase from 8.39 to 8.63, to $8.82 \AA^{2} / \mathrm{fs}$, respectively. When the 'combustion' ReaxFF force field was implemented, the self-diffusion coefficient increased from 7.44 to 7.54 , to $7.8810^{-4} \AA^{2} / \mathrm{fs}$, 
respectively. Note that the correspondent simulation box size increased from $32.1 \times 32.1 \times 32.1$ $\AA^{3}$, to $40.4 \times 40.4 \times 40.4 \AA^{3}$, to $46.3 \times 46.3 \times 46.3 \AA^{3}$, respectively. These self-diffusion coefficients, calculated from both ReaxFF and TraPPE models, are shown in Figure 7 as a function of the inverse box size $(1 / \mathrm{L})$. The data are fitted using a straight line and used to extrapolate the $\mathrm{CO}_{2}$ self-diffusion coefficient at infinite box size. These results confirm that $D_{s}$ increases with the box size for all systems considered. Quantifying for all systems considered here the thermodynamic limit for $D_{S}$ is beyond the scopes of the present manuscript. Because somewhat similar system size effects are observed for both classic and reactive force fields, such effects do not affect the conclusions of this manuscript.

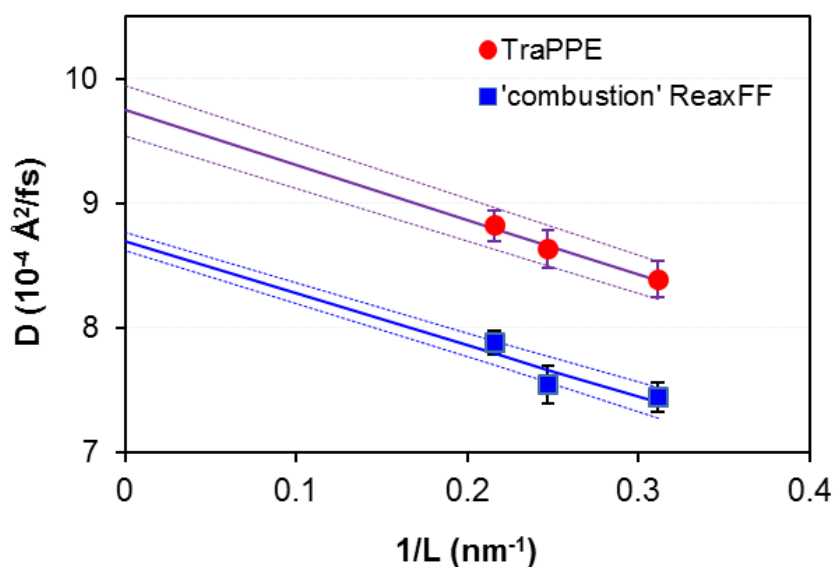

Figure 7. Diffusion coefficient of liquid $\mathrm{CO}_{2}$ as a function of the inverse box size 1/L. Symbols are simulation data, continuous lines are fits to the data and extrapolations to the infinite box size. The Error bars are obtained as one standard deviation from the mean of at least 3 simulation runs. The dotted lines highlight the uncertainty in these extrapolations.

Implementing 'combustion' ReaxFF yields self-diffusion coefficients for $\mathrm{CO}_{2}$ in both liquid and gaseous phases that are in reasonable agreement with the non-reactive force field, and also with available experimental data. 'Combustion' ReaxFF yields reasonable self-diffusion coefficients for gaseous $\mathrm{CH}_{4}$ and $\mathrm{H}_{2}$, while it yields self-diffusion coefficients for liquid $\mathrm{CH}_{4}$ twice as fast as the values predicted by the non-reactive simulations, and for liquid $\mathrm{H}_{2}$ about 9 times slower than the values predicted by the non-reactive simulations. In the case of water, we report in Table 6 results obtained using both 'combustion' and 'aqueous' ReaxFF parameterisations. The 'combustion' ReaxFF version under-predicts the self-diffusion coefficient of both vapour and liquid water (by a factor of $\sim 1500$ and $\sim 5$, respectively), compared to classical force fields, while the 'aqueous' ReaxFF version provides excellent predictions for the self-diffusion coefficient of liquid water and gaseous water. Raju et al. [15] reported that the self-diffusion coefficient of bulk liquid water at $298 \mathrm{~K}$ is $\sim 2.1110^{-4}$ $\AA^{2} / \mathrm{fs}$, which is in reasonable agreement with our results, as well as with experiments [77-78]. 
TABLE 6. Self-diffusion coefficients as predicted by all models implemented in this work. For comparison, experimental data are also reported.

\begin{tabular}{|c|c|c|c|c|c|}
\hline \multirow{2}{*}{ Molecule } & \multirow{2}{*}{$\begin{array}{l}\text { Temperature } \\
(\mathrm{K})\end{array}$} & \multirow{2}{*}{ Force field } & \multicolumn{3}{|c|}{ Self-diffusion coefficient } \\
\hline & & & This study & Experiment & Unit \\
\hline \multirow{6}{*}{$\mathrm{CH}_{4}$} & \multirow{3}{*}{150} & TraPPE & $4.40 \pm 0.13$ & \multirow{3}{*}{$6.06^{\mathrm{a}}$} & \multirow{3}{*}{$10^{-4} \AA^{2} / \mathrm{fs}$} \\
\hline & & OPLS & $6.15 \pm 0.11$ & & \\
\hline & & 'combustion' ReaxFF & $9.39 \pm 0.24$ & & \\
\hline & \multirow{3}{*}{298} & TraPPE & $2.46 \pm 0.12$ & \multirow{3}{*}{$2.34^{\mathrm{b}}$} & \multirow{3}{*}{$\AA^{2} / \mathrm{fs}$} \\
\hline & & OPLS & $2.02 \pm 0.02$ & & \\
\hline & & 'combustion' ReaxFF & $1.93 \pm 0.02$ & & \\
\hline \multirow{8}{*}{$\mathrm{CO}_{2}$} & \multirow{4}{*}{273} & TraPPE & $8.39 \pm 0.15$ & \multirow{4}{*}{$8.42^{\mathrm{c}}$} & \multirow{4}{*}{$10^{-4} \AA^{2} / \mathrm{fs}$} \\
\hline & & Zhang & $9.57 \pm 0.32$ & & \\
\hline & & EPM2 & $9.13 \pm 0.27$ & & \\
\hline & & 'combustion' ReaxFF & $7.44 \pm 0.12$ & & \\
\hline & \multirow{4}{*}{298} & TraPPE & $1.32 \pm 0.13$ & \multirow{4}{*}{$1.13^{\mathrm{b}}$} & \\
\hline & & Zhang & $1.24 \pm 0.18$ & & \\
\hline & & EPM2 & $1.35 \pm 0.11$ & & \\
\hline & & 'combustion' ReaxFF & $1.20 \pm 0.07$ & & \\
\hline \multirow{6}{*}{$\mathrm{H}_{2} \mathrm{O}$} & \multirow{3}{*}{298} & $\mathrm{SPC} / \mathrm{E}$ & $2.40 \pm 0.17$ & \multirow{3}{*}{$2.30^{\mathrm{d}}$} & \multirow{3}{*}{$10^{-4} \AA^{2} / \mathrm{fs}$} \\
\hline & & 'aqueous' ReaxFF & $2.39 \pm 0.05$ & & \\
\hline & & 'combustion' ReaxFF & $0.52 \pm 0.04$ & & \\
\hline & \multirow{3}{*}{500} & SPC & $1.01 \pm 0.02$ & \multirow{3}{*}{ - } & \multirow{3}{*}{$\AA^{2} / \mathrm{fs}$} \\
\hline & & 'aqueous' ReaxFF & $1.04 \pm 0.05$ & & \\
\hline & & 'combustion' ReaxFF & $\begin{array}{c}0.66 \pm 0.08 \\
\left(10^{-3}\right)\end{array}$ & & \\
\hline \multirow{4}{*}{$\mathrm{H}_{2}$} & \multirow[t]{2}{*}{15.2} & Frost & $3.88 \pm 0.07$ & \multirow{2}{*}{$4.50^{\mathrm{e}}$} & \multirow{2}{*}{$10^{-4} \AA^{2} / \mathrm{fs}$} \\
\hline & & 'combustion' ReaxFF & $0.41 \pm 0.05$ & & \\
\hline & \multirow{2}{*}{77.5} & Frost & $1.38 \pm 0.14$ & \multirow{2}{*}{$1.33^{\mathrm{f}}$} & \multirow{2}{*}{$\AA^{2} / \mathrm{fs}$} \\
\hline & & ‘combustion' ReaxFF & $1.29 \pm 0.02$ & & \\
\hline
\end{tabular}

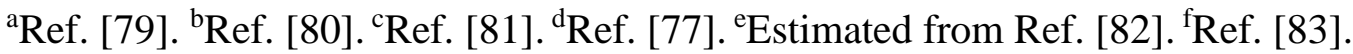

\section{DISCUSSION and CONCLUSIONS}

In this study we calculated structural and dynamic properties of four common pure fluids by conducting equilibrium molecular dynamics simulations implementing the reactive and nonreactive force fields. The 'combustion' ReaxFF was considered, as well as several classical non-reactive force fields widely used to study fluids. The comparison is limited to radial distribution functions, which are related to the ability of a model to predict configurational thermodynamic properties of a fluid, and self-diffusion coefficients, which are related to the ability of a model to predict transport properties for a fluid. We observed several results in agreement, but also significant differences among the predictions when reactive and nonreactive force fields are implemented. In the liquid phase, experimental data, when available, are in good agreement with the classic simulations, suggesting that the classic models could 
be good approximations for the properties of pure fluids. For liquid hydrogen, however, neither classical nor ReaxFF simulations reproduce the expected structure. In the gas phase, the second virial coefficient was used to assess the ability of the simulations to reproduce experimental data. Experimental data are indeed available for the second virial coefficient of gases, and this quantity can be readily calculated from radial distribution function datasets.

It can be seen from the site-site correlation functions for $\mathrm{CH}_{4}$ molecules in Fig. 1, that the configuration of liquid molecules within the first solvation shell predicted by the 'combustion' ReaxFF calculation is similar to that predicted by TraPPE and OPLS models. Meanwhile, a significant variation is observed between 'combustion' ReaxFF and OPLS results for $\mathrm{CH}_{4}$ in the gas phase. Comparing the predicted second virial coefficients to experimental data, we conclude that a realistic $\mathrm{g}_{\mathrm{CC}}$ should be in between that obtained from the 'combustion' ReaxFF and that obtained from the OPLS non-reactive simulations. All the results for the radial distribution function for liquid methane show a small shift towards shorter distances. A similar trend was also found for gas-phase molecules. The 'combustion' ReaxFF simulations yield a greater self-diffusion coefficient for liquid $\mathrm{CH}_{4}$ compared to both OPLS and TraPPE models as well as to experimental data. The 'combustion' ReaxFF gasphase self-diffusion coefficient $\mathrm{CH}_{4}$ is slightly smaller than that obtained from TraPPE simulations and that measured experimentally, but it is consistent with OPSL model simulations. Thus, we conclude that the 'combustion' ReaxFF yields acceptable values for the diffusivity of pure $\mathrm{CH}_{4}$ in vapour phases.

The pair correlation functions shown in Fig. 2 and Fig. 3 suggest that 'combustion' ReaxFF simulations are in slightly better agreement with classic simulations regarding the spatial arrangement of gas-phase $\mathrm{CO}_{2}$ molecules rather than that of liquid-phase ones. However, the results for the second virial coefficients suggest that none of the force fields implemented yield a sufficiently attractive effective interaction between the $\mathrm{CO}_{2}$ molecules in the gas phase. In all cases, it was found that 'combustion' ReaxFF calculations yield peak positions shifted to slightly longer distances compared to the respective classical simulations. It was also found that the 'combustion' ReaxFF simulations yield transport properties (i.e., selfdiffusion coefficients) for both liquid and gaseous $\mathrm{CO}_{2}$ that are in reasonable agreement with classical simulations as well as with experiments.

The 'combustion' ReaxFF simulations yield structures for pure $\mathrm{H}_{2}$ that are in reasonable agreement with classical simulations, albeit the agreement is better for the gaseous phase compared to liquid $\mathrm{H}_{2}$. In fact, the classic model employed here was derived to study gaseous hydrogen. In the liquid phase both 'combustion' ReaxFF and classical simulations yield radial distribution functions that differ substantially compared to theoretical predictions from the literature, most likely because quantum effects are not considered either in the Frost model or in the 'combustion' ReaxFF model. The results for the self-diffusion coefficients show that 'combustion' ReaxFF predictions are reasonable for the gaseous phase, but rather poor for the liquid phase. 

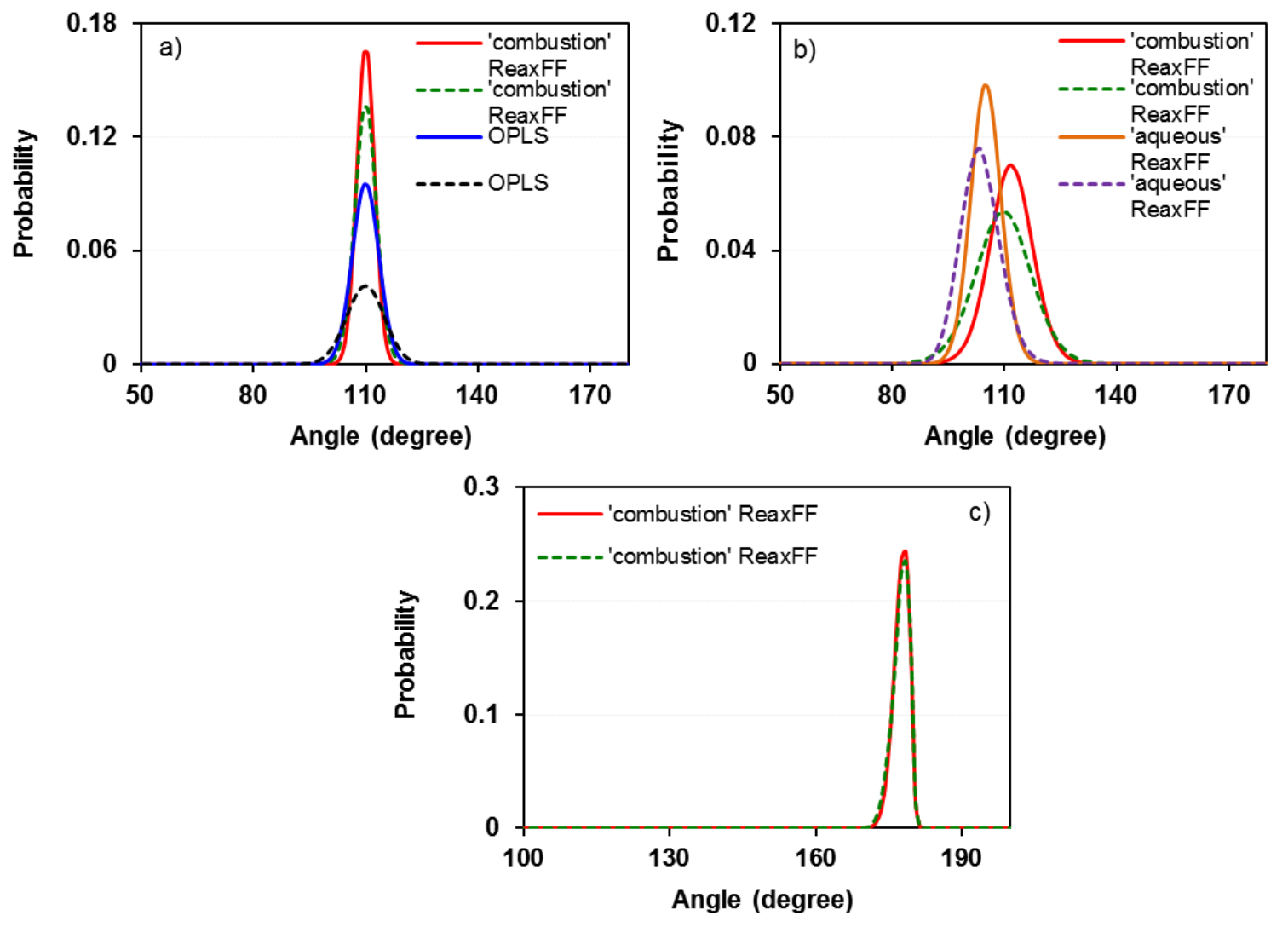

Figure 8. Probability density distribution for the angle for molecules studied using 'combustion' ReaxFF, 'aqueous' ReaxFF and OPLS model: $\mathrm{CH}_{4}(\mathrm{a}) \mathrm{H}_{2} \mathrm{O}$ (b) and $\mathrm{CO}_{2}$ (c).

Solid and dashed lines represent molecules in liquid and gas phase, respectively.

To explain the differences just summarized between 'combustion' ReaxFF and classical simulations we first consider the bond length and bond angle parameters as implemented in the various force fields. It is worth pointing out that all classical force field models considered, except OPLS, are rigid, while in ReaxFF simulations both bond lengths and angles can vary during a simulation. In Figure 8 we quantify the probability density distribution of the $\mathrm{H}-\mathrm{C}-\mathrm{H}$ angle in $\mathrm{CH}_{4}$, the $\mathrm{H}-\mathrm{O}-\mathrm{H}$ angle in $\mathrm{H}_{2} \mathrm{O}$, and the O-C-O angle in $\mathrm{CO}_{2}$ as obtained for ReaxFF simulations and OPLS simulations. The results show that, compared to the expected geometries of the simulated molecules, 'combustion' ReaxFF simulations predict smaller O-C-O angles in $\mathrm{CO}_{2}\left(178.5^{\circ}\right.$ for both the liquid and the gas), similar angles for $\mathrm{CH}_{4}\left(109.5^{\circ}\right.$ for both liquid and gas) and larger angles for $\mathrm{H}_{2} \mathrm{O}\left(109.5^{\circ}\right.$ in the gas phase and $115.5^{\circ}$ in the liquid phase). Perhaps these differences explain the ability, or lack thereof, of the 'combustion' ReaxFF parameterization to estimate structure and dynamics of the pure fluids considered here.

Regarding water, our results show that the 'combustion' ReaxFF yields unrealistic predictions for the properties of bulk water, both in the liquid and in the vapour phases. However, the 'aqueous' ReaxFF parameterization yields a structure for pure water that is in very good agreement with that predicted by classical force fields, and self-diffusion coefficients that are also in very good agreement with classical simulations. The ReaxFF 
parameter sets are classified into two major groups (i.e., the ReaxFF branches), intratransferable with one another: the combustion versus the aqueous branch. The $\mathrm{O} / \mathrm{H}$ parameters proposed by these two data sets differ significantly because the combustion branch focuses on accurately describing water as a gas-phase molecule, while the aqueous branch focuses on reproducing aqueous chemistry. 'Combustion' ReaxFF was originally developed for applications at temperatures above the water boiling point, where combustion reactions typically occur. On the other hand, the $\mathrm{O} / \mathrm{H}$ parameters from 'aqueous' ReaxFF were developed to describe liquid water, thus explaining the success of 'aqueous' ReaxFF simulations in reproducing SPC/E simulation results. It is somewhat unexpected that, based on our simulation results, the 'combustion' ReaxFF parameterization yields poor predictions for structure and diffusion of water even in the gaseous phase. As shown in Figure 5 this is may be due to water association, suggesting that the temperature is still too low for 'combustion' ReaxFF simulations of water to be successful.

In conclusion, comparing reactive and classical simulations we found that the ReaxFF parameterisation can be helpful at predicting structure and dynamics of pure fluids, but the agreement with both classical simulations and experiments depends strongly on the fluid considered and on the thermodynamics conditions simulated. It is encouraging that the 'aqueous' ReaxFF parameterization yields results in excellent agreement with those predicted using some of the most widely implemented models for bulk water.

\section{ACKNOWLEDGMENTS}

We acknowledge the financial support from the U.S. Department of Energy, Office of Basic Energy Sciences, under Contract No. DE-SC0006878 (Division of Chemical Sciences, Geosciences, and Biosciences), Geosciences Program. Additional financial support was provided by the A. P. Sloan Foundation via the Deep Carbon Observatory administered by the Carnegie Institution for Science. AS acknowledges financial support from the European Union via the Marie Curie Career Integration Grant No. 2013-CIG-631435. Generous allocations of computing time were provided by the National Energy Research Scientific Computing Center (NERSC) at Lawrence Berkeley National Laboratory, Berkeley, CA. NERSC is supported by the DOE Office of Science. We are also grateful to the Oklahoma Supercomputer Center for Education and Research (OSCER), for access to high-performance computing. We acknowledge the suggestions from one of the anonymous reviewers, who, among other important comments, reminded us that experimental second virial coefficients could be used to compare against simulation results.

\section{References}

[1] Martin MG, Siepmann JI. Transferable potentials for phase equilibria. 1. United-atom description of n-alkanes. J Phys Chem B. 1998;102:2569-2577.

[2] Martin MG, Siepmann JI. Novel configurational-bias Monte Carlo method for branched molecules. Transferable potentials for phase equilibria. 2. United-atom description of branched alkanes. J Phys Chem B. 1999;103:4508-4517. 
[3] Wick CD, Martin MG, Siepmann JI. Transferable potentials for phase equilibria. 4. United-atom description of linear and branched alkenes and alkylbenzenes. J Phys Chem B. 2000;104:8008-8016.

[4] Chen B, Potoff JJ, Siepmann JI. Monte Carlo calculations for alcohols and their mixtures with alkanes. Transferable potentials for phase equilibria. 5. United-atom description of primary, secondary, and tertiary alcohols. J Phys Chem B. 2001;105:3093-3104.

[5] Lubna N, Kamath G, Potoff JJ, et al. Transferable potentials for phase equilibria. 8. United-atom description for thiols, sulfides, disulfides, and thiophene. J Phys Chem B. 2005;109:24100-24107.

[6] Senftle TP, Hong S, Islam MM, et al. The ReaxFF reactive force-field: development, applications and future directions. npj Comput Mater. 2016;2:15011.

[7] Han Y, Jiang D, Zhang J, et al. Development, applications and challenges of ReaxFF reactive force field in molecular simulations. Front Chem Sci Eng. 2016;10:16-38.

[8] Chenoweth K, van Duin ACT, Goddard WA. ReaxFF reactive force field for molecular dynamics simulations of hydrocarbon oxidation. J Phys Chem A. 2008;112:1040-1053.

[9] Nielson KD, van Duin ACT, Oxgaard J, et al. Development of the ReaxFF reactive force field for describing transition metal catalyzed reactions, with application to the initial stages of the catalytic formation of carbon nanotubes. J Phys Chem A. 2005;109:493-499.

[10] Zou C, Van Duin A. Investigation of complex iron surface catalytic chemistry using the ReaxFF reactive force field method. Jom-Us. 2012;64:1426-1437.

[11] Shin YK, Kwak H, Vasenkov AV, et al. Development of a ReaxFF reactive force field for $\mathrm{Fe} / \mathrm{Cr} / \mathrm{O} / \mathrm{S}$ and application to oxidation of butane over a pyrite-covered $\mathrm{Cr}_{2} \mathrm{O}_{3}$ catalyst. Acs Catal. 2015;5:7226-7236.

[12] Goddard III W, Merinov B, Van Duin A, et al. Multi-paradigm multi-scale simulations for fuel cell catalysts and membranes. Mol Simul. 2006;32:251-268.

[13] Bedrov D, Smith GD, van Duin ACT. Reactions of singly-reduced ethylene carbonate in lithium battery electrolytes: a molecular dynamics simulation study using the ReaxFF. J Phys Chem A. 2012;116:2978-2985.

[14] Le T, Striolo A, Turner CH, et al. Confinement effects on carbon dioxide methanation: a novel mechanism for abiotic methane formation. Sci Rep. 2017;7:9021.

[15] Raju M, Kim S-Y, van Duin ACT, et al. ReaxFF reactive force field study of the dissociation of water on titania surfaces. J Phys Chem C. 2013;117:10558-10572.

[16] Huang L, Gubbins KE, Li L, et al. Water on titanium dioxide surface: a revisiting by reactive molecular dynamics simulations. Langmuir. 2014;30:14832-14840.

[17] Aimoli CG, Maginn EJ, Abreu CRA. Transport properties of carbon dioxide and methane from molecular dynamics simulations. J Chem Phys. 2014;141:134101.

[18] van Duin ACT, Dasgupta S, Lorant F, et al. ReaxFF: A reactive force field for hydrocarbons. J Phys Chem A. 2001;105:9396-9409.

[19] Page AJ, Moghtaderi B. Molecular dynamics simulation of the low-temperature partial oxidation of $\mathrm{CH}_{4}$. J Phys Chem A. 2009;113:1539-1547.

[20] He Z, Li X-B, Liu L-M, et al. The intrinsic mechanism of methane oxidation under explosion condition: a combined ReaxFF and DFT study. Fuel. 2014;124:85-90.

[21] He Z-H, Li X-B, Zhu W-J, et al. Effect of water on gas explosions: combined ReaxFF and ab initio MD calculations. Rsc Adv. 2014;4:35048-35054.

[22] Cheng T, Jaramillo-Botero A, Goddard III WA, et al. Adaptive accelerated ReaxFF reactive dynamics with validation from simulating hydrogen combustion. J Am Chem Soc. 2014;136:9434-9442.

[23] Plimpton S. Fast parallel algorithms for short-range molecular-dynamics. J Comput Phys. 1995;117:1-19. 
[24] Aktulga HM, Fogarty JC, Pandit SA, et al. Parallel reactive molecular dynamics: numerical methods and algorithmic techniques. Parallel Comput. 2012;38:245-259.

[25] Leach AR. Molecular modelling: principles and applications. Prentice Hall; 2001.

[26] Jorgensen WL, Maxwell DS, Tirado-Rives J. Development and testing of the OPLS allatom force field on conformational energetics and properties of organic liquids. J Am Chem Soc. 1996;118:11225-11236.

[27] Harris JG, Yung KH. Carbon dioxide's liquid-vapor coexistence curve and critical properties as predicted by a simple molecular model. J Phys Chem 1995;99:12021-12024.

[28] Potoff JJ, Siepmann JI. Vapor-liquid equilibria of mixtures containing alkanes, carbon dioxide, and nitrogen. Aiche J. 2001;47:1676-1682.

[29] Zhang Z, Duan Z. An optimized molecular potential for carbon dioxide. J Chem Phys. 2005;122:214507.

[30] Berendsen HJC, Postma JPM, van Gunsteren WF, et al. Interaction models for water in relation to protein hydration. In: Pullman B, editor. In Intermolecular forces: Proceedings of the fourteenth Jerusalem symposium on quantum chemistry and biochemistry held in Jerusalem, Israel, April 13-16, 1981. Dordrecht: Springer Netherlands; 1981, pp. 331-342.

[31] Jorgensen WL, Chandrasekhar J, Madura JD, et al. Comparison of simple potential functions for simulating liquid water. J Phys Chem. 1983;79:926-935.

[32] Berendsen HJC, Grigera JR, Straatsma TP. The missing term in pffective pair potentials. J Phys Chem 1987;91:6269-6271.

[33] Glättli A, Daura X, Van Gunsteren WF. A novel approach for designing simple point charge models for liquid water with three interaction sites. J Comput Chem. 2003;24:10871096.

[34] Abascal JLF, Sanz E, Fernández RG, et al. A potential model for the study of ices and amorphous water: TIP4P/Ice. J Chem Phys. 2005;122:234511.

[35] Abascal JLF, Vega C. A general purpose model for the condensed phases of water: TIP4P/2005. J Chem Phys. 2005; 123:234505.

[36] Vega C, Abascal JLF, Sanz E, et al. Can simple models describe the phase diagram of water? J Phys-Condens Mat. 2005; 17:S3283-S3288.

[37] Vega C, Abascal JLF, Conde MM, et al. What ice can teach us about water interactions: a critical comparison of the performance of different water models. Faraday Discuss. 2009; 141:251-276.

[38] Soper AK. The radial distribution functions of water and ice from 220 to $673 \mathrm{~K}$ and at pressures up to $400 \mathrm{MPa}$. Chem Phys. 2000;258:121-137.

[39] Guillot B. A reappraisal of what we have learnt during three decades of computer simulations on water. J Mol Liq. 2002;101:219-260.

[40] Cummings PT, Cochran HD, Simonson JM, et al. Simulation of supercritical water and of supercritical aqueous-solutions. J Chem Phys. 1991;94:5606-5621.

[41] Chialvo AA, Cummings PT. Engineering a simple polarizable model for the molecular simulation of water applicable over wide ranges of state conditions. J Chem Phys. 1996;105:8274-8281.

[42] Darkrim F, Vermesse J, Malbrunot P, et al. Monte Carlo simulations of nitrogen and hydrogen physisorption at high pressures and room temperature. Comparison with experiments. J Chem Phys. 1999;110:4020-4027.

[43] Hirschfelder JO, Curtis CF and Bird RB. Molecular theory of gases and liquids. Wiley: New York; 1954.

[44] Cracknell RF. Molecular simulation of hydrogen adsorption in graphitic nanofibres. Phys Chem Chem Phys. 2001;3:2091-2097.

[45] Yang Q, Zhong C. Molecular simulation of adsorption and diffusion of hydrogen in metal-organic frameworks. J Phys Chem B. 2005;109:11862-11864. 
[46] Salles F, Kolokolov DI, Jobic H, et al. Adsorption and diffusion of $\mathrm{H}_{2}$ in the MOF type systems MIL-47(V) and MIL-53(Cr): a combination of microcalorimetry and QENS xxperiments with molecular simulations. J Phys Chem C. 2009;113:7802-7812.

[47] Frost H, Düren T, Snurr RQ. Effects of surface area, free volume, and heat of adsorption on hydrogen uptake in metal-organic frameworks. J Phys Chem B. 2006;110:9565-9570.

[48] Michels A, de Graaff W, Ten Seldam CA. Virial coefficients of hydrogen and deuterium at temperatures between $-175^{\circ} \mathrm{C}$ and $+150^{\circ} \mathrm{C}$. Conclusions from the second virial coefficient with regards to the intermolecular potential. Physica. 1960;26:393-408.

[49] Kinaci A, Haskins JB, Çağin T. On calculation of thermal conductivity from Einstein relation in equilibrium molecular dynamics. J Chem Phys. 2012;137:014106.

[50] Vrij A, Tuinier, R. Fundamentals of interface and colloid science, Vol IV. Elsevier: Amsterdam; 2005.

[51] Essmann U, Perera L, Berkowitz ML, et al. A smooth particle mesh ewald method. J Chem Phys. 1995;103:8577-8593.

[52] Allen MP, Tildesley DJ. Computer simulation of liquids. Oxford University Press: Oxford; 2004.

[53] Friend DG, Ely JF, Ingham H. Thermophysical properties of methane. J Phys Chem Ref Data. 1989;18:583-638.

[54] Suehiro Y, Nakajima M, Yamada K. Critical parameters of $\left\{\mathrm{xCO}_{2}+(1-\mathrm{x}) \mathrm{CHF}_{3}\right\}$ for $\mathrm{x}=(1.0000,0.7496,0.5013$, and 0.2522). J Chem Thermodyn. 1996;28:1153-1164.

[55] Sato H, Watanabe K, Sengers JMHL, et al. Sixteen thousand evaluated experimental thermodynamic property data for water and steam. J Phys Chem Ref Data. 1991;20:10231044.

[56] Reid R, Prausnitz JM, Poling BE. The properties of gases and liquids (4th ed.). McGraw Hill, New York; 1987.

[57] Stassen H. On the pair potential in dense fluid methane. J Mol Struct: Theochem. 1999;464:107-119.

[58] Straus $\beta$ G, Bassen A, Zweier H, et al. High-pressure neutron diffraction on fluid methane. Phys Rev E. 1996;53:3505-3517.

[59] Murad S, Evans DJ, Gubbins KE, et al. Molecular-dynamics simulation of dense fluid methane. Mol Phys. 1979;37:725-736.

[60] Williams DE. Nonbonded potential parameters derived from crystalline hydrocarbons. J Chem Phys. 1967;47:4680-4684.

[61] van Tricht JB, Fredrikze H, van der Laan J. Neutron diffraction study of liquid carbon dioxide at two thermodynamic states. Mol Phys. 1984;52:115-127.

[62] Monti S, van Duin ACT, Kim S-Y, et al. Exploration of the conformational and reactive dynamics of glycine and diglycine on $\mathrm{TiO}_{2}$ : computational investigations in the gas phase and in solution. J Phys Chem C. 2012;116:5141-5150.

[63] Lindenau T, Ristig ML, Gernoth KA, et al. The physics of liquid para-hydrogen. Int J Mod Phys B. 2006;20:5035-5046.

[64] Dawidowski J, Bermejo FJ, Ristig ML, et al. Static structure factor of liquid parahydrogen. Phys Rev B. 2004;69:014207.

[65] Singh JK, Kofke DA. Mayer sampling: Calculation of cluster integrals using free-energy perturbation methods. Phys Rev Lett. 2004;92:220601.

[66] Schultz AJ, Kofke DA, Harvey AH. Molecular-based virial coefficients of $\mathrm{CO}_{2}-\mathrm{H}_{2} \mathrm{O}$ mixtures. Aiche J. 2015;61:3029-3037.

[67] Yang S, Schultz AJ, Kofke DA. Evaluation of second and third dielectric virial coefficients for non-polarisable molecular models. Mol Phys. 2017;115:991-1003.

[68] Smith DE, Haymet ADJ. Free energy, entropy, and internal energy of hydrophobic interactions: Computer simulations. J Chem Phys. 1993;98:6445-6454. 
[69] Koga K, Widom B. Thermodynamic functions as correlation-function integrals. J Chem Phys. 2013;138:114504.

[70] Widom B, Underwood RC. Second osmotic virial coefficient from the two-component van der Waals equation of state. J Phys Chem B. 2012;116:9492-9499.

[71] Koga K, Holten V, Widom B. Deriving second osmotic virial coefficients from equations of state and from experiment. J Phys Chem B. 2015;119:13391-13397.

[72] Ohgaki K, Mizuhaya T, Katayama T. The interaction virial coefficient for seven binary systems containng carbon dioxide, methane, ethylene, ethane and propylene at $25^{\circ} \mathrm{C}$. $\mathrm{J}$ Chem Eng Japan. 1981;14:71-72.

[73] Dadson RS, Evans EJ, King JH. The second virial coefficient of carbon dioxide. Proc Phys Soc. 1967;92:1115-1121.

[74] Harvey AH, Lemmon EW. Correlation for the second virial coefficient of water. J Phys Chem Ref Data. 2004;33:369-376.

[75] Goodwin RD, Diller DE, Roder HM, et al. Second and third virial coefficients for hydrogen. J Res Natl Bur Std. 1964;68A:121-126.

[76] Yeh I-C, Hummer G. System-size dependence of diffusion coefficients and viscosities from molecular dynamics simulations with periodic boundary conditions. J Phys Chem B. 2004;108:15873-15879.

[77] Mills R. Self-diffusion in normal and heavy water in the range 1-45.deg. J Phys Chem. 1973;77:685-688.

[78] Holz M, Heil SR, Sacco A. Temperature-dependent self-diffusion coefficients of water and six selected molecular liquids for calibration in accurate H-1 NMR PFG measurements. Phys Chem Chem Phys. 2000;2:4740-4742.

[79] Greiner-Schmid A, Wappmann S, Has M, et al. Self-diffusion in the compressed fluid lower alkanes - methane, ethane, and propane. J Chem Phys. 1991;94:5643-5649.

[80] Winn EB. The temperature dependence of the self-diffusion coefficients of argon, neon, nitrogen, oxygen, carbon dioxide, and methane. Phys Rev. 1950;80:1024-1027.

[81] Gros $\beta$ T, Buchhauser J, Lüdemann HD. Self-diffusion in fluid carbon dioxide at high pressures. J Chem Phys. 1998;109:4518-4522.

[82] Souers PC. Hydrogen properties for fusion energy. University of California Press; 1986.

[83] Mason EA, Annis BK, Islam M. Diffusion coefficients of $\mathrm{T}_{2}-\mathrm{H}_{2}$ and $\mathrm{T}_{2}-\mathrm{D}_{2}$ : the nonequivalence of $\mathrm{H}_{2}$ and $\mathrm{D}_{2}$ cross sections. J Chem Phys. 1965;42:3364-3366. 